\title{
Traumatic brain injuries
}

Kaj Blennow ${ }^{1,2}$, Dave Brody ${ }^{3}$, Patrick M. Kochanek ${ }^{4}$, Harvey Levin ${ }^{5,6}$, Ann McKee ${ }^{7}$, Gerard M. Ribbers ${ }^{8}$, Kristine Yaffe ${ }^{9}$, Henrik Zetterberg ${ }^{1,2,10}$

Correspondence to K.B.

E-mail: kaj.blennow@neuro.gu.se

Clinical Neurochemistry Laboratory

Institute of Neuroscience and Physiology

The Sahlgrenska Academy at University of Gothenburg

Sahlgrenska University Hospital, Mölndal

SE-43180 Mölndal, Sweden

\section{Author addresses}

1) Institute of Neuroscience and Physiology, Department of Psychiatry and Neurochemistry, the Sahlgrenska Academy at the University of Gothenburg, Mölndal, Sweden;

2) Clinical Neurochemistry Laboratory, Sahlgrenska University Hospital, Mölndal, Sweden;

3) Dept. of Neurology, Washington University, St. Louis School of Medicine, St. Louis, MO, USA;

4) Dept. of Critical Care Medicine, Safar Center for Resuscitation Research, Univ. of Pittsburgh School of Medicine, Pittsburgh, PA, USA;

5) Dept. of Physical Medicine and Rehabilitation, Baylor College of Medicine, Houston, TX, USA;

6) Michael E. De Bakey Veterans Affairs Medical Center, Houston.

7) Dept. of Neurology, Boston University School of Medicine, Boston, MA, USA;

8) Dept. of Rehabilitation Medicine and Physical Therapy, Erasmus MC, University Medical Center Rotterdam, and Rijndam Rehabilitation Center, Rotterdam, The Netherlands;

9) Depts. of Psychiatry, Neurology, Epidemiology and Biostatistics, University of California, San Francisco; Department of Veterans Affairs, San Francisco Veterans Affairs Medical Center, San Francisco, CA, USA;

10) UCL Institute of Neurology, Queen Square, London, United Kingdom 


\title{
Competing interests
}

KB has served on advisory boards for IBL International, and Roche Diagnostics and provided consultation to Fujirebio Europe, and is a confounder together with $\mathrm{HZ}$ a cofounder of Brain Biomarker Solutions in Gothenburg AB, a GU Venture-based platform company at the University of Gothenburg. PMK, DB, HL, AM, GMR and KY declare no competing interests.

\begin{abstract}
Traumatic brain injury (TBI) is clinically divided into a spectrum of severities, with mild TBI being the least severe form. Mild TBI is caused by blunt non-penetrating head trauma that causes stretching and tearing of axons, the long neuronal extensions, with diffuse axonal injury (DAI) being the central pathogenic mechanism. Mild TBI is basically synonymous with concussion; both are defined on pure clinical grounds in different consensus criteria that are largely compatible. Symptoms in mTBI are highly variable; loss of consciousness may, or may not, be present. There are no validated imaging or fluid biomarkers to identify (or rule out) whether a patient with a normal CT scan has neuronal damage. While most patients recover quickly from mTBI, others report persistent symptoms, a condition called post-concussive syndrome (PCS). The pathophysiology underlying PCS, and the relative importance of the severity of neuronal damage and psychosocial factors for its development, are largely unknown. Repeated concussive and sub-concussive head injuries have been linked to the neurodegenerative condition chronic traumatic encephalopathy (CTE), which has been reported in a series of contact sports athletes sports and in some soldiers exposed to explosive blasts. Hyperphosphorylation and aggregation of the neuronal protein tau is suggested as the key pathological finding in CTE. Modern MRI techniques to identify and grade axonal injury and PET methods for tau and amyloid pathology show promise as diagnostic tools for CTE. Longitudinal clinical studies employing such techniques are needed to learn what percentage of contact sport athletes will develop CTE, and what number and severity of concussive events that are required for developing this disorder. Given that CTE mainly affects contact sport athletes, and is believed to be due to repetitive head trauma, unique opportunities for prevention by rule changes are open for discussion by sports organizations and legislators.
\end{abstract}

\section{[H1] Introduction}


Traumatic brain injury (TBI) affects people of all ages and is recognized as a major cause of death and disability worldwide ${ }^{1}$. TBI can be categorized into mild, moderate and severe, largely based on clinical variables such as duration of unconsciousness (if present), amnesia and neurological symptoms ${ }^{2}$ (Box 1). Moderate and severe TBI are neurosurgical and intensive care concerns; the focus of this Primer article is concussion or mild TBI, which represent $80-90 \%$ of all cases ${ }^{3}$, and how mild TBI may increase the risk of longterm and sometimes progressive neurologic disease.

Mild TBI and concussion are interchangeable terms for the mildest form of TBI, both defined purely on clinical grounds, and conceptualized in clinical criteria outlined by different working groups, that largely are compatible with each other. ${ }^{3-5}$ In short, mild TBI is typically caused by blunt non-penetrating head trauma and results in transient symptoms. ${ }^{6}$ Symptoms (clinical observations and often patient self-reporting) in mild TBI are highly variable, and can, except for loss of consciousness (not a prerequisite), include physical (e.g., nausea and vomiting, dizziness, headache), cognitive (e.g., poor concentration and memory problems), and behavioral (e.g., irritability, and emotional lability) symptoms ${ }^{4}$. According to the American Congress of Rehabilitation Medicine (ACRM) criteria, ${ }^{4}$ computerized tomography $(\mathrm{CT})$ of the brain, as well as a routine neurological examination, may (or may not) be normal. ${ }^{4}$ loss of consciousness (if present) should be less than 30 min, at which time the Glasgow Coma Scale (GSC) score should be 13-15, and posttraumatic amnesia (if present) should not exceed 24 hours, for a TBI to be classified as mild. It is generally agreed across criteria that there is no single test to assist making a clinical diagnosis of mild TBI.

Mild TBI ${ }^{5}$ symptoms resolve within 7-10 days in the majority (80-90\%) of cases. Most patients who exhibit post-concussive symptoms following a mild TBI show symptom resolution within 1-12 weeks. The recovery period after sports concussion may be shorter since athletes typically are in better physical condition than patients evacuated to emergency centers for non-sports mild TBI, who more frequently have pre-existing behavioral, psychiatric, and/or substance abuse problems that increase their susceptibility to injury and may prolong recovery ${ }^{6,7}$, although an alternative explanation may be that sports-related TBIs result from lower mechanical forces. However, those who have persisting symptoms are said to have post-concussive syndrome (PCS) ${ }^{8}$. The symptoms of 
PCS are highly variable (Box 2), which also makes its exact prevalence difficult to estimate; $10-15 \%$ is probably a reasonable inference ${ }^{8}$.

Chronic traumatic encephalopathy (CTE) has garnered much recent attention as a longterm consequence of repeated mTBIs and repetitive sub-concussive head impacts, particularly in contact sport athletes and military veterans. First described by Martland ${ }^{9}$ in 1928 as "punch drunk syndrome" in retired boxers, the condition has also been called "dementia pugilistica"10 before being referred to as CTE in the $1940 \mathrm{~s}^{11-13}$.

Neuropathologically, CTE has been associated with widespread neurofibrillary tangles in several brain regions ${ }^{14}$. Widespread amyloid plaques, similar to those in Alzheimer's disease, may be found in approximately $50 \%$ of CTE cases, and are significantly associated with age and the inheritance of the apolipoprotein $\mathrm{E}(A P O E) \varepsilon 4$ allele $\mathrm{e}^{15}$. _ENREF_17In 2005, the first case report on CTE in a former American football player was published $^{16}$, and after that CTE pathology has been identified in several other contact sports, such as ice hockey, wrestling and rugby, and also in soldiers exposed to explosive blasts, although the number of cases is limited ${ }^{17}$. At present, CTE is a neuropathological diagnosis requiring post-mortem examination, given that there are no established or validated clinical criteria and no biomarkers to support the diagnosis ante mortem.

In this Primer article, we give a comprehensive update on mild TBI and concussion in adults, together with the relation to its possible sub-acute and long-term consequences, PCS and CTE. In this review, we regard TBI as a spectrum of severities, with mild TBI being the least intense, instead of clear-cut entities. An exception is the addition of other types of injuries (contusions, bleedings) in the severe forms, due to the more extensive mechanical forces involved. We find it unlikely that there is any pathophysiologic distinction between the different TBI severities, and instead plausible that the same underlying cellular and molecular processes are involved, just to different degrees.

\section{[H1] Epidemiology}

TBI is a leading cause of disability and death with almost 10 million people affected worldwide each year ${ }^{18}$. A recent meta-analysis from 16 European countries estimates that the incidence of hospital-admitted TBI cases is 262 per $100,000 .{ }^{19}$. The Centers for Disease 
Control and Prevention in the United States report an increase from 567 to 824 per 100,000 TBI-related emergency department visits between 2000 and $2010^{20}$. The incidence of TBI has increased in middle and low-income countries. ${ }^{21}$ The true incidence of TBI is likely higher given that mild TBI cases, which probably account for $70-90 \%$ of all cases, are underestimated in most studies ${ }^{1}$. A large statewide population-based study reported that more than $40 \%$ of participants had at least one TBI during lifetime. ${ }^{22}$

The most common causes of TBI of any severity are falls and traffic injuries, which together account for more than $50 \%$ of cases. ${ }^{19,20,23}$ Rates of TBI tend to be higher among men compared to women with the peak incidence among adults in the oldest age groups. ${ }^{19}$, ${ }^{20,23}$ Surveillance data suggests that the incidence of sports-related TBI visits to emergency rooms in the USA is 152 per 100,000, with almost two-thirds occurring in children and young adults $<19$ years of age, with numbers growing in both males and females. ${ }^{24}$ In addition, military service members represent a unique risk group.

Epidemiological studies show that negative outcomes (disability, poor life satisfaction, and memory impairment) increase in prevalence with increasing TBI severity. ${ }^{22}$ Such studies also suggest an association between TBI and risk of dementia. A meta-analysis of 15 casecontrol studies reported an increased odds ratio (OR) of 1.58 (95\% CI 1.21-2.06) for the association between head injury and risk of Alzheimer's disease. ${ }^{25}{ }^{26-28}$ However, it should be noted that these studies are based on clinically diagnosed Alzheimer's disease patients, which is known to be unreliable also in memory clinic settings; the studies did not include autopsy or biomarker (amyloid PET or CSF A $\beta$ and tau) assessed cases. Therefore, the

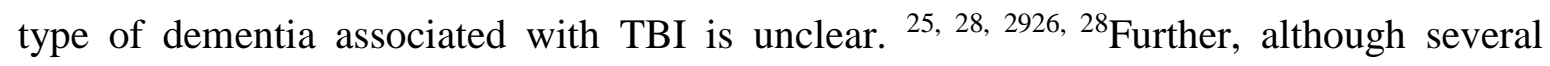
studies have demonstrated a dose-response effect such that risk of dementia increases with increasing severity of $\mathrm{TBI},{ }^{26,} 27$ the risk associated with mild $\mathrm{TBI}$ is unknown. ${ }^{28}$ Investigation of mild TBI effects may be particularly difficult as mild TBIs are underreported and the definition of mild TBI is often not uniform across studies. ${ }^{28}$

Epidemiological evidence is also incomplete for the risks associated with repetitive TBIs and CTE. Although cognitive and behavioral changes are reported among contact sports athletes who experience repetitive head injuries, ${ }^{29}$ there are no estimates for prevalence or incidence. A limitation is that no imaging or fluid biomarkers are available to identify CTE 
clinically, and differentiate this disorder from $\mathrm{AD}$ or other neurodegenerative diseases. ${ }^{18}$, 31

\section{[H1] Mechanisms/pathophysiology}

\section{[H2] Biophysical mechanisms}

Improved understanding of how the physical forces from an external blow to the head are transmitted and transduced in the brain should shed light on how physiological functions are affected and pathogenic processes of TBI are initiated.

Brain damage in TBI is caused by rotational (angular) and/or linear (translational) acceleration forces or by blunt trauma with impact deceleration (Figure 1). Such acceleration and deceleration forces generate intracranial pressure gradients through the inertia of the brain that lags behind the skull during the rapid movement. As a result, axons are stretched and damaged leading to diffuse axonal injury (DAI) ${ }^{30}$. Whether different types of acceleration and deceleration forces cause axonal damage by different mechanisms is unclear; either focal strains (because the brain cannot rotate within the skull) or strains owing to differences in densities between brain tissues at different sites (e.g. the cortex and the underlying white matter) might be at play ${ }^{31}$. Indeed, biomechanical studies show that morphologic heterogeneity of the cortex is important in diffuse brain injury; higher stress and strain forces are evident in the bottom of the sulci in the cerebral cortex $^{32}$, matching tau pathology in CTE, which is localized to these cortical structures ${ }^{33}$.

Exactly how the shearing forces cause the axonal damage is not known. However, the severity of DAI is directly proportional to the severity of brain injury as measured by the severity of symptoms and functional outcomes, and occurs after brain acceleration/deceleration forces are applied - not secondary to other types of injury such as ischemia or inflammation ${ }^{34}$. Concussions in contact sports are also related to the forces of the head impacts. In American football, concussions are related to the degree of head acceleration from the impacts ${ }^{35}$. In boxing, the rotational acceleration due to a punch increases in heavier weight class $^{36}$, which is easy to imagine given that it has been estimated that a punch by a professional boxer generates the same force on impact as being hit in the head by a $6 \mathrm{~kg}$ bowling ball rolling at $20 \mathrm{mph}^{37}$. 


\section{[H2] Neuropathology}

\section{[H3] Pathology of acute mild TBI}

Neuropathological changes found after concussion and other forms of mild TBI include mild multifocal axonal injury, microglial activation and microhaemorrhages. ${ }^{38,} 39$ The multifocal axonal injury typically involves the fornices, corpus callosum, subcortical white matter and cerebellum. ${ }^{40}$ Less consistent findings include TDP-43 immunopositive neurites, whereas cytoplasmic neuronal TDP-43 inclusions are not found until later stages, and small focal accumulations of paired helical filament (PHF)-tau, either as NFTs or neuropil threads. ${ }^{40}$

\section{[H3] Pathology of mild CTE}

Neuropathology of CTE includes both gross alterations, with cavum septum pellucidum or septal fenestrations being common pathological findings, and microscopical changes in the form of accumulation of (PHF)-tau aggregates in neurons and astroglia, neurofibrillary tangle formation, astrocytic inclusions and dot-like and spindle shaped neurites. ${ }^{33}$ Tau abnormalities are most often distributed around small blood vessels at the depths of cortical sulci and in an irregular pattern, and have a tendency to involve the superficial cortical layers (Figure 2). ${ }^{33}$ Indeed, this pathognomonic lesion distinguishes CTE from other neurodegenerative tauopathies, including Alzheimer's disease, progressive supranuclear palsy, agyrophilic grain disease, corticobasal degeneration, primary agerelated tauopathy (PART), parkinsonism dementia complex of Guam (GPDC) and nonspecific tau astrogliopathy (ARTAG). ${ }^{39,41}$ _ENREF_45A staging scheme of progressive PHF-tau pathology with four stages (stage I-IV) has been proposed that will be important for future studies on CTE prevalence and its relation to number and severity of repeated TBIs. $^{42}$

Other abnormalities in CTE include deposits of phosphorylated 43-kDa TAR DNAbinding protein (TDP-43) protein in the majority (80\%) of cases that occasionally colocalized with PHF-tau, axonal dystrophy and neuroinflammation. ${ }^{42,43}$ In addition, around half of CTE cases have deposition of amyloid $\square(\mathrm{A} \beta)$ as either diffuse or neuritic plaques, and a proportion $(14 \%)$ meet the criteria for Alzheimer's disease. ${ }^{15}$ When compared to an autopsy community cohort, the deposition of $\mathrm{A} \beta$ was altered and accelerated in CTE such that CTE subjects were overall 4-fold more likely to have $\mathrm{A} \beta$ plaques and developed 
plaques 10-15 years earlier. Moreover, $A \beta$ plaques were significantly associated with more severe PHF-tau and Lewy body pathology and worse clinical outcome independent of the effect of age.

Recent neuropathological studies indicate that CTE pathology may be common among contact sport athletes. ${ }^{44}$ However, further studies are necessary to clarify what proportion of athletes develop this type of pathology and how many become symptomatic, as well as how many athletes escape CTE pathology despite repeated concussions.

\section{[H2] Cellular and molecular pathophysiology}

\section{[H3] Axonal injury}

Studies of the molecular pathophysiology of TBI have examined many domains including neuronal cell death, astroglial injury, astroglial and microglial responses, dendritic injury, and synaptic dysfunction. Notably, though, much of the field has focused on the topic of axonal injury (Figure 3), largely because of clinical and experimental observations linking the severity of axonal injury to the extent of disability following $\mathrm{TBI}^{45}$. For example, axonal injury is consistently revealed by amyloid precursor protein (APP) immunohistochemistry (APP undergoes fast axonal transport and accumulates at sites of axonal injury with transport failure) in every TBI of all severities, ranging from concussion (with death from other causes) to severe $\mathrm{TBI}{ }^{46,47}$. In a non-human primate TBI model, the depth of coma following rotational acceleration injury was directly proportional to the extent of axonal injury in the brainstem ${ }^{34}$. Similar observations were subsequently reported in a pig model ${ }^{48}$. Using non-invasive imaging methods such as diffusion tensor MRI and fluid biomarker-based measurements of axonal injury-related proteins, several investigators have demonstrated correlation between axonal injury severity and outcomes in patients with mild TBI and in patients with more-severe injuries. ${ }^{49}, 50$ Thus, the focus on axonal injury seems to be well justified by the clinical literature.

Axonal injury can be detected immediately after injury when forces of sufficient magnitude are applied to the brain. Electron microscopic analyses indicate that microtubules are disrupted within 2 minutes after a rapid stretch of the axons of cultured neurons ${ }^{51}$. Subsequently, axonal varicosities (distorted and irregular axonal swellings or local dilations) form, apparently due to failed axonal transport along the disrupted 
microtubules. Indeed, these varicosities are the most common form of axonal injury and show accumulation of APP due to disturbed axonal transport. Similar axonal varicosities are a hallmark of traumatic axonal injury observed after TBI in human patients. ${ }^{52,53}$ Axons in general, and microtubules specifically, may be more mechanically brittle than other structures in the nervous system, although this idea has not been rigorously tested. Dynamic instability may also contribute to microtubule disruption and failed axonal transport in $\mathrm{TBI}^{51}$.

Subsequent investigations have revealed other forms of axonal injury ${ }^{54,55}$. For example, cytoskeletal neurofilament compaction has been observed by electron microscopy and immunohistochemistry using antibodies recognizing neurofilament neo-epitopes ${ }^{56,57}$. The mechanisms underlying this neurofilament compaction include disruption of the integrity of the plasma membrane leading to increased intracellular calcium, activation of calpain and caspase, and cytoskeletal proteolysis including spectrin and neurofilament side arm cleavage. ${ }^{58}$ Another form of axonal injury involves collapse, rather than dilation. Such

injury has been detected by electron microscopy after experimental concussion ${ }^{59}$. The mechanisms underlying axoplasmic collapse remain to be determined.

Importantly, not all forms of axonal injury result in disconnection of axons ${ }^{60}$; injured axons can remain connected at least over the time span of the observations, although whether these injured but still connected axons are functional remains to be determined. Notably, the extent of traumatic axonal injury can be greatly underestimated in studies based on conventional thick-section light microscopic histopathology, which is only capable of visualizing dilated axons and axonal varicosities. Non-dilated, injured axons cannot be resolved with conventional histopathology, but instead require electron microscopy or ultrathin section-based approaches ${ }^{61}$. To our knowledge, super-resolution light microscopic studies of injured axons have not yet been reported.

\section{[H3] Amyloid $\square$ accumulation}

$\mathrm{A} \beta$ is a key component of plaques in Alzheimer's disease, and is produced by sequential cleavage from APP by $\beta$-secretase (BACE1) and the $\gamma$-secretase complex (with presenilin constituting the active site) ${ }^{62}$ Epidemiological studies suggest a link between TBI and risk of late life dementia in general, and of Alzheimer's disease in particular. There are two 
major hypotheses regarding the mechanisms underlying this increased risk $^{63}$; the first is that TBI reduces cognitive reserve such that the same pathophysiological processes underlying Alzheimer's disease cause detectible symptoms at an earlier age, leading to a net increase in prevalence of dementia. The second hypothesis is that TBI directly sets into motion the tau and $\mathrm{A} \beta$ pathophysiological processes underlying Alzheimer's disease. These two hypotheses are not mutually exclusive.

Evidence to support the accelerated $A \beta$ hypothesis includes the findings of diffuse $A \beta$ plaques in a subset of human patients with severe TBI at younger ages than would be expected. ${ }^{64,} 65$ Experimentally, TBI in pigs causes diffuse A $\square$ plaques, and intra-axonal deposition of $A \beta^{66}$. The origin of the extracellular plaques is unknown; microdialysisbased studies in human and mice indicate that soluble extracellular A $\square$ does not increase, but instead decreases acutely after TBI and then recovers as neurological function is restored, likely due to recovery of synaptic activity ${ }^{67,68}$. A similar recovery phase may explain the delayed rise in $A \beta$ observed in ventricular cerebrospinal fluid in patients with acute $\mathrm{TBI}^{69}$. In contrast, acutely elevated $\mathrm{A} \beta$ levels have been observed in tissue lysates following experimental injury ${ }^{70}$; the reasons for this discrepancy may have to do with partial mixing of increased intracellular and decreased extracellular $\mathrm{A} \beta$ pools in tissue lysates $^{68}$. The increase in intra-axonal $A \beta$ is believed to arise from the aberrant colocalization of APP, the $\gamma$-secretase complex, and the $\beta$-secretase BACE1 at the sites of failed axonal transport ${ }^{71}$. Inhibition of $\gamma$-secretase prevents TBI-related intra-axonal A $\beta$ formation in mice, but does not seem to affect the overall severity of axonal injury ${ }^{72}$. Recent work on the pathophysiology of age-related Alzheimer's disease has emphasized the role of soluble oligomeric assemblies of $A \beta^{73,74}$ as key mediators of the synaptic dysfunction and memory loss that underlie Alzheimer's disease ${ }^{75-78}$. However, the effects of TBI on A $\beta$ oligomers remain to be determined. Thus, TBI has complex and incompletely characterized effects on $\mathrm{A} \beta$.

\section{[H3] Tau aggregation}

Tau aggregation has been observed in a subset of TBI patients, most commonly in those with CTE. Tau is a microtubule-stabilizing protein, and it has been hypothesized that upregulation of tau may be an aberrant attempt to stabilize microtubules disrupted by the trauma. An alternative hypothesis is that aberrant phosphorylation of tau leads to loss of its 
normal microtubule-binding function and promotes its aggregation. In other contexts, A $\beta$ aggregates may induce tau phosphorylation and aggregation ${ }^{79}$; however, in the setting of experimental TBI, 90\% reduction in $\mathrm{A} \beta$ levels had no effect on TBI-related tau aggregation ${ }^{72}$. In contrast, c-jun N-terminal kinase (JNK) activation was co-localized with tau aggregates, and JNK inhibition reduced TBI-related tau aggregation ${ }^{80}$. Thus, the mechanisms underlying TBI-related tau aggregation may be distinct from the mechanisms elucidated in other contexts.

\section{[H3] Other types of pathophysiological changes}

The inflammatory responses to TBI are major pathophysiological events that may affect outcomes in complex ways. Microglia, the resident innate immune cells in the brain, are dramatically activated in the first days to a week after injury and can either be transient or persistent. ${ }^{81,82}$ However, the mechanisms underlying microglial activation, their effects on debris removal, repair, recovery, progressive degeneration and long-term neurodegenerative sequelae remain to be determined ${ }^{83}$. Furthermore, the current categorization of microglia into M1 "classically activated" and M2 "alternatively activated" sub-types may be inadequate for characterization of responses to TBI ${ }^{84}$. Soluble cytokine response is also quite dramatic in human TBI patients ${ }^{85}$, further supporting that inflammation may be a therapeutic target, at least in severe TBI ${ }^{86}$. After TBI, free radical stress in the form of high levels of oxidized and nitrosylated proteins, lipids and nucleic acids can be detected in the brain. Early free radical stress may be due to e.g. the arachidonic acid cascade, mitochondrial dysfunction, and nitric oxide synthase activation in severely injured portions of the brain ${ }^{87}$ but is not as clearly involved in less severely injured areas or in concussive injury. Specific mitochondrial lipid oxidation has recently been demonstrated, and may play an important role in apoptotic cell death ${ }^{88}$. The effects of delayed free radical stress from microglial and other inflammatory cellular responses is just beginning to be explored ${ }^{89}$. In summary, the role of inflammation in TBI warrants further study, especially to elucidate what are the various roles for microglial activation after TBI, and which roles are beneficial and which are harmful, with regard to clearance of debris and promoting recovery.

\section{[H2]Genetic factors}


Genetic factors may influence both short-term survival and long-term neurological and functional outcome after TBI by affecting several different putative pathogenic pathways. Short-term survival may depend on gene variants influencing the severity of axonal damage, or other processes such as inflammation or blood-brain barrier disruption, whereas long-term outcome may depend on genes involved in neuronal regeneration and plasticity. Some studies have found associations between polymorphisms in genes involved in neuronal plasticity, e.g. the brain derived neurotrophic factor (BDNF) gene, and inflammatory response, e.g. the interleukin $(I L)$ genes ${ }^{90}$. In general, studies examining potential associations between gene polymorphisms and TBI outcome are hampered by difficulties in dividing cohorts with highly variable brain damage into groups with comparable injuries due to the lack of biomarkers to measure the severity of neuronal damage, and also that outcome is measured using relatively crude rating scales or other outcome measures.

The most examined susceptibility gene for short and long-term prognosis after TBI is the apolipoprotein $\mathrm{E}$ gene $(A P O E)$. The $A P O E$ gene encodes for a protein involved in cholesterol and lipid metabolism, and is a main component of plasma lipoproteins. ApoE is also the principal apolipoprotein in the CNS, where it is involved in neuronal repair through recycling of cell membrane lipids to build new cell neuronal membranes, neurites and synapses during regeneration in response to brain injury ${ }^{91,92}$.

The $A P O E$ gene is polymorphic, with three alleles called $A P O E \varepsilon 2, \varepsilon 3$, and $\varepsilon 4$, with the $\varepsilon 3$ allele being most common in the population. The APOE $\varepsilon 4$ allele is a well-established and strong susceptibility gene for Alzheimer's disease ${ }^{62}$, affecting both disease risk and age at onset, but has also been suggested to influence prognosis or rate of progression of several other brain disorders ${ }^{93}$. An association between poor short-term clinical outcome after TBI and the $A P O E \& 4$ allele has been found in some studies, but associations are weak and variable and several studies report no effect ${ }^{90}$. Preclinical studies on experimental TBI suggest that ApoE may affect the severity of axonal injury; mice with the $\varepsilon 4$ allele showed larger numbers of dilated, APP stained axons than $\varepsilon 2$ or $\varepsilon 3$ mice $^{94}$. Further, several clinical studies suggest that possession of the APOE $\varepsilon 4$ allele predicts poor long-term (6-12 months) clinical outcome after TBI, as also verified in a recent meta-analysis that showed an effect especially in severe TBI cases ${ }^{95}$. 
Studies examining the $A P O E$ gene in relation to CTE are scarce. The first indication for an association was a study on retired professional boxers with high exposure to head-blows in which $\varepsilon 4$ carriers had more severe symptoms of chronic brain injury ${ }^{96}$. Further support for an effect of the APOE gene on CTE risk include a study finding lower cognitive performance in older football players carrying the APOE $\varepsilon 4$ allele ${ }^{97}$, and a small neuropathological series of $10 \mathrm{CTE}$ cases showing a high (50\%) proportion of APOE $\varepsilon 4$ carriers $^{33}$. The mechanisms underlying the putative association between the APOE gene and CTE risk may involve isoform-dependent effects on neuronal regeneration and plasticity or may be linked to build-up of tau or A $\beta$ pathology. In Alzheimer's disease, the leading hypothesis is that $A \beta$ aggregation and clearance are differentially regulated by the ApoE isoforms ${ }^{98}$. Considering that amyloid plaques are found only in later stage CTE cases $^{33}$, mechanisms related to $\beta$-amyloidosis are unlikely to be involved in initiating the disease. Further large clinical studies applying consensus criteria for the clinical diagnosis of CTE as well as biomarkers to subgroup cases with brain amyloidosis and tauopathy are warranted to verify and explore the mechanisms for the association between APOE and CTE, and to identify novel risk genes.

\section{[H1] Diagnosis, screening and prevention}

\section{[H2] TBI severity}

Acute TBI severity is classified primarily by level of consciousness measured by the lowest post-resuscitation Glasgow Coma Scale (GCS) score ${ }^{99}$. This score consists of graded responses to behaviors (eye opening, and verbal and motor responses to questions and pain stimuli). However, since some components of the GCS scale may be untestable (if, for example, the patient is intubated) or cannot be tested reliably, clinicians are recommended to report subscale scores for individual patients ${ }^{100}$. For use in the clinical setting, clinicians should, however, consider brain imaging, neurophysiological measures, other neurological findings such a pupillary response, and fluid biomarkers in addition to the GCS score in classification of TBI, monitoring clinical management, and prognosis ${ }^{100}$.

\section{[H2]Clinical characteristics of PCS and CTE}

Patients having symptoms persisting several months after mTBI are classified as having post-concussive syndrome (PCS). However, PCS is a difficult diagnosis since there are no objective diagnostic tools. In addition, PCS symptoms, such as headache, fatigue, sleep 
disturbances and anxiety, are common in everyday life in healthy people, especially in persons with pre-existing psychiatric conditions such as anxiety and depression. Further, symptoms are influenced by personality and psychological ${ }^{8}$ and demographic factors such as social status, sex, ethnicity and alcohol use, ${ }^{101}$ that also may affect rate of recovery after mild TBI.

CTE is also ambiguous to diagnose clinically, due to varying clinical features and lack of established biomarkers to support a clinical suspicion of the disease. Clinical criteria for the diagnosis of traumatic encephalopathy syndrome (TES), the clinical counterpart of CTE, have been defined and are currently undergoing evaluation in a large longitudinal study ${ }^{102}$.

Individuals who develop CTE tend to have begun their sport early, and while symptoms at varying ages, one third are symptomatic at the time they retire from the sport, and in most cases the condition advanced slowly over several decades ${ }^{33}$. Retrospective descriptions of symptoms suggest three primary subtypes: behavioral/mood changes (irritability, depression, sleep disturbance), cognitive deficits (poor attention, memory problems, executive dysfunction, dementia) and a mixed feature subtype ${ }^{102}$. Motor deficits (Parkinsonism, ataxia, dysphasia) have also been described in former boxers with the dementia pugilistica CTE variant ${ }^{14}$ __Pending results of current prospective, longitudinal studies of at-risk athletes with a control group, these descriptions should be interpreted as preliminary, for further details on sports-related concussion, see Box 3.

\section{[H3]Endocrine complications}

The most common endocrine complications following TBI are related to injury to the pituitary gland. The gland itself is well protected within the sella turcica of the sphenoid bone; however, the pituitary stalk, connected to the anterior pituitary gland and hypothalamus, is vulnerable to the mechanical forces of TBI. Post-traumatic hypopituitarism (PTHP) is found in around 30\% of TBI patients, with higher prevalence in more severe cases ${ }^{103}$. The most common abnormalities are growth hormone $(\mathrm{GH})$ deficiency and hypogonadism, whilst adrenocorticotrophic hormone (ACTH) and thyroidstimulating hormone (TSH) deficiencies are diagnosed less often ${ }^{104,105}$. Almost $50 \%$ of TBI patients have anti-diuretic hormone deficiency severe enough to cause central diabetes 
insipidus in the acute phase following the injury, but this deficiency often resolves within 3 months ${ }^{105,106}$.

PTHP can result in a multitude of acute and chronic symptoms ${ }^{107}$. For example, GH deficiency may result in changes in body composition and a reduction in quality of life including depression, whilst hypothyroidism may cause weight gain, low mood and blunted cognition ${ }^{107}$. Most crucially, acute adrenal insufficiency may occur within days of TBI in some patients, with the ensuing adverse consequences of hypotension, hyponatraemia and hypoglycaemia, which require emergent treatment. PTHP is also relevant in the context of mild TBI; a recent study demonstrated a prevalence of GH deficiency of $1 \%$ in a cohort of patients with a history of concussion ${ }^{108}$ and even higher prevalence has been reported in athletes with a history of repetitive head trauma ${ }^{107}$. For example, high prevalence (25-30\%) of hypopituitarism with GH and ACTH deficiency has been reported in amateur kick boxers and retired American football players that may result in poor quality of life, erectile dysfunction and metabolic syndrome ${ }^{109,110}$. Likewise, as many as $42 \%$ of military personnel with previous blast-related mTBI had abnormal hormone levels in one or more pituitary axes ${ }^{111}$. Given the subtle nature of some of the symptoms, screening strategies in patients with a history of TBI were recently proposed ${ }^{112}$, 113.

\section{[H2]Imaging}

CT scans are fast, accurately detect essentially all life-threatening and surgically treatable intracranial hemorrhages in TBI patients ${ }^{114}$. However, CT scans do not detect axonal injury and provide only modest information regarding prognosis. Indeed, even if CT is part of the diagnostic criteria, many patients with mild TBI evaluated in the emergency department and most cases of sports concussion do not undergo any structural brain imaging. Further, it has been reported that $28 \%$ of patients with normal CT have lesions on MRI performed in the research setting within 2 weeks of the trauma, with lesions (contusions and/or multiple foci of hemorrhagic axonal injury) being associated with worse outcome ${ }^{115}$.

\section{[H3] MRI-based techniques}

MRI scans provide superior anatomical detail over CT, more sensitive detection of smaller hemorrhages, and improved information regarding prognosis ${ }^{114-116}$. However, MRI is less clinically available and has longer scan times. Further, conventional MRI sequences 
provide little ability to resolve axonal injury (Figure 4), and most concussive TBI patients have normal conventional MRI scans, even though they often have other evidence of traumatic axonal injury. For this reason, extensive efforts have gone into developing improved MRI sequences that are more sensitive to axonal injury.

Diffusion tensor imaging (DTI) is the leading MRI sequence for the detection of axonal injury (Figure 4). ${ }^{50,117}$ DTI measures diffusion of water in many directions, and from these measurements, the directional asymmetry, termed anisotropy, of water diffusion can be calculated. Injured axons have lower anisotropy, and in experimental animal the extent of reduced anisotropy quantitatively correlates strongly with the extent of axonal injury measured by immunohistochemistry. ${ }^{114,118}$ Reduced anisotropy after TBI has been reported in a large number of clinical studies of patients with many types of TBI, at a variety of time points after injury even when CT and conventional MRI showed no evidence of injury. ${ }^{117,119}$ Reductions in anisotropy in specific white matter tracts was found to correlate with TBI-related cognitive deficits ${ }^{120}$, and to the neuronal protein tau measured in microdialysis fluid ${ }^{121}$. Limitations of DTI include its relative insensitivity to injury in complex white matter regions, inconsistencies in the literature concerning the direction of change in anisotropy after mTBI, and lack of spatial resolution to detect injury in small white matter tracts ${ }^{122}$. Furthermore, DTI needs standardization across different scanners, making it necessary to obtain control data on each scanner for each study. Novel diffusion imaging methods such as diffusion kurtosis imaging ${ }^{123}$, high angular resolution diffusion imaging ${ }^{124}$, high definition fiber tracking ${ }^{125}$, and diffusion spectrum imaging ${ }^{126}$ may be even more sensitive to axonal injury. Magnetic resonance spectroscopy is capable of resolving alterations in brain metabolism after TBI, and good correlations between metabolite concentrations and outcomes have been reported. ${ }^{127,}{ }^{128}$ Functional MRI (fMRI) scans also reveal changes after TBI, with areas of either reduced or increased activation, and altered connectivity. ${ }^{129}$

Magnetoencephalography (MEG) detects changes in magnetic fields caused by brain electrical activity. Time resolution of MEG is orders of magnitude better than fMRI, allowing resolution of fast activity and transient changes in functional connectivity. MEG may detect abnormalities following TBI that were not identified with DTI or conventional MRI, and may correlate with clinical outcome. ${ }^{130,131}{ }^{130-133}$. However, MEG is available 
only at a few centers, similar to DTI has limited spatial resolution, and cannot yet be readily standardized between sites.

\section{[H3]PET}

PET ligands have been developed that bind to aggregated $A \beta$ and give a measure of the amount of amyloid deposits in different brain regions as compared with cerebellum that is used as a reference region. Alzheimer's disease patients typically show a 50-70\% increased ligand retention in cortical brain regions as compared that found in elderly controls ${ }^{134}$. Except for the ${ }^{11} \mathrm{C}$-labelled amyloid ligand $\mathrm{PiB}$, which has a short half-life that hinders its use outside specialized research centers with access to an on-site cyclotron, ${ }^{18} \mathrm{~F}$-labeled tracers with longer half-lives, including florbetapir, flutemetamol and florbetaben, have been developed for a more wide-spread use of amyloid PET.

A first pilot study using $\mathrm{PiB}$ amyloid PET showed young to middle-aged patients with moderate to severe TBI had increased amyloid deposition in cortical grey matter and in the striatum as compared with controls in the acute period up to 1 year post-trauma ${ }^{135}$. Another small study on severe TBI cases examined 11-17 months after injury showed increased PiB retention in the precuneus/posterior cingulate and cerebellum ${ }^{136}$. Amyloid deposition in the posterior cingulate correlated with decreasing fractional anisotropy assessed by DTI, but was much lower than the amount found in Alzheimer's disease cases ${ }^{136}$. There are no studies on amyloid PET in cases with mild TBI.

PET using 18F-labeled fluorodeoxyglucose can measure glucose uptake and metabolism in different brain regions. Perturbed brain FDG-PET is found in Alzheimer's disease, other neurodegenerative disorders, and psychiatric disease such as schizophrenia and bipolar disorders. ${ }^{137}$ The general trend for an outcome in moderate to severe TBI studies is increased glucose metabolism in some brain regions during the acute phase, followed by a prolonged hypometabolism during the sub-acute to chronic phase, while mild TBI is less studied and findings variable. ${ }^{138}$

Recent developments have given several tau PET tracers with high binding affinity and selectivity to PHF-tau deposits in the brain ${ }^{139}$. Tau PET is currently being evaluated in Alzheimer's disease, a show promise to monitor the development of tau pathology during the clinical course of the disease and to improve understanding of pathophysiology ${ }^{140}$. 
However, there are no clinical studies on tau PET in TBI or CTE patients. Large longitudinal clinical studies on TBI patients and cases with PCS and suspected CTE will be important to learn how frequent amyloid and tau deposition are, during which phase after TBI these proteins deposits develop, and how they correlate with other measures of neuronal damage and with clinical symptoms, disease stage and prognosis.

\section{[H2]Fluid biomarkers}

Fluid biomarkers are objective measures of pathogenic process linked to a disease or an injury. For acute TBI, biomarkers can be used to grade severity of brain damage and predict prognosis, guide clinical management, monitor therapeutic interventions, and in sports-related TBI to guide return-to-play decisions. Biomarker fluids of relevance to TBI include cerebrospinal fluid (CSF), serum and plasma. An optimal biomarker for TBI should reflect the pathophysiological processes.

\section{[H3] Cerebrospinal fluid biomarkers}

CSF sampling through a ventricular catheter in patients in need of intracranial pressure monitoring is used in clinical proof-of-concept studies on severe TBI, to evaluate candidate CSF biomarkers for different pathophysiological processes and their performance to predict survival or long-term functional outcome. Results from such studies may guide the design of CSF biomarker studies on mild TBI. The high sensitivity of CSF biomarkers to detect even minor brain damage, in patients with negative CT/MRI scans, may give important knowledge on the pathophysiology of mild TBI. It may be argued that lumbar puncture will be difficult to implement in the clinic in the evaluation of patients with acute mTBI. This has been the case for CSF biomarker for early diagnosis of Alzheimer's disease, but increasing scientific data supporting their high performance also for early diagnosis have gradually changed the situation so that these diagnostic tools are increasingly used for diagnostic purposes in clinical practice. ${ }^{141}$

Increased CSF/serum albumin ratio, a standard biomarker of blood-brain barrier function, has been reported in the context of a neuroinflammatory response in patients with severe TBI ${ }^{142,143}$. In mild TBI, no such changes have been seen ${ }^{144,145}$, suggesting that the BBB remains intact or only 'opens up' transiently in mild TBI. 
A large number of studies report an acute phase response within the central nervous system following severe TBI, which is reflected in the CSF as increased concentrations of proinflammatory proteins such as IL-6, IL-8 and IL-10. ${ }^{146,147}$

The best-established CSF biomarkers for axonal injury are total-tau (T-tau) and neurofilament light (NF-L; a structural protein mainly expressed in large caliber myelinated axons) ${ }^{148}$. Studies using ELISA show that T-tau concentrations in ventricular CSF correlate with lesion size and outcome in severe TBI, so that high levels indicate worse injury. ${ }^{148,149} \mathrm{~A}$ recent pilot study using the enhanced immunoassay using multiarrayed fiberoptics (EIMAF) technique found increases in ventricular CSF in both T-tau and P-tau during the acute phase in a small series of severe TBI cases ${ }^{150}$. Studies on mild TBI show elevated CSF concentrations of both T-tau and NF-L but the magnitude of the rise is larger for NF-L than for T-tau suggesting that blows to the head impact long, myelinated axons in the white matter more than short, non-myelinated axons in the cortex, which is in agreement with experimental, as well as neuroimaging and neuropathological studies ${ }^{145,151}$.

NSE is a glycolytic enzyme enriched in neuronal cell bodies ${ }^{152}$. NSE concentrations are higher in ventricular CSF of non-survivors following severe TBI than in survivors, and/or correlate with other TBI severity scores in both adults and children. ${ }^{153,}{ }^{154}$ The major limitation of CSF NSE as a biomarker for neuronal injury is its high concentration in erythrocytes that may contaminate the sample ${ }^{155}$.

$\mathrm{S}-100 \mathrm{~B}$, an astrocyte-enriched $\mathrm{Ca}^{2+}$-binding protein has been examined extensively in relation to TBI in serum, while studies on CSF are rare. Amateur boxers have slightly elevated CSF levels after one or several bouts, but the changes are not as pronounced as those for the axonal markers T-tau and NF-L ${ }^{151}$. Similar results have been reported for glial fibrillary acidic protein (GFAP) ${ }^{145,151}$, which is an intermediate filament that is almost exclusively expressed in astrocytes ${ }^{152}$. However, CSF GFAP has also been evaluated in the context of severe TBI and ventricular levels have been shown to improve outcome prediction models in conjunction with clinical data ${ }^{156}$. Further, high GFAP levels in blood samples may predict clinically relevant intracranial lesions in TBI patients. ${ }^{157}$ 
Studies in both animals and humans have demonstrated that APP and A $\beta$ accumulate in neurons and axons after brain trauma with axonal damage ${ }^{12}$. Ventricular CSF and brain interstitial fluid concentrations of the 40 and 42 amino acid isoforms of $A \beta$ increase during the first week of brain injury in severe $\mathrm{TBI}^{69,158}$ and particularly in diffuse axonal injury ${ }^{159}$. Similar results have been obtained for soluble isoforms of APP ${ }^{69}$. However, in studies of mild TBI performed on lumbar CSF samples, no amyloid-related changes have been seen ${ }^{145,151}$.

The protein $\alpha$ II-spectrin is found in neuronal axons and presynaptic terminals in the brain 160. $\alpha$ II-spectrin is proteolytically processed to $\alpha$ II-spectrin breakdown products (SBDPs) that have been suggested as potential TBI biomarkers both in animal experimental studies and in humans 161,162 . SBDPs have been analyzed in ventricular CSF from patients with severe TBI and the levels correlate with clinical correlates of the severity of the injury and predict outcome ${ }^{156,163,164}$. In one of these studies, CSF SBDPs were evaluated together with another proposed TBI biomarker, ubiquitin C-terminal hydrolase-L1 (UCHL1) ${ }^{156}$, which is a de-ubiquitinating enzyme highly expressed in neurons ${ }^{165}$. The two markers were found to contribute clinically relevant information in addition to routine assessments.

\section{[H3]Blood biomarkers}

Based on the above, candidate blood biomarkers for TBI include NSE, UCHL1, S100B, GFAP, spectrin N-terminal fragments (SNTF), T-tau and NF-L. N-terminal fragments of $\alpha$-spectrin (SNTF) accumulate in damaged axons following $\mathrm{TBI}^{166}$.

Blood levels of GFAP and UCHL1 have in several studies been found to have high power to discriminate between patients with TBI and controls. (Diaz 2014; Nylen 2006). ${ }^{167,168}$ Further, higher UCHL1 levels are found in TBI patients with abnormal CT scans, with an AUC value of 0.67 for UCHL1, ${ }^{167}$ and 0.88 for GFAP. ${ }^{169}$ A recent very large study on mild-to-moderate TBI showed AUC values of 0.80-0.97 for GFAP to detect intracranial lesions on CT and of 0.91-1.00 to identify patients in need of neurosurgical interventions, while performance was lower for UCHL1, ${ }^{170}$ another study showed similar performance to identify abnormal CT in mild-to-moderate TBI cases similar for all of FGAP, UCHL1 and S100B. ${ }^{171}$ 
There are few studies on the performance of these biomarkers in mild TBI cases, but one study found higher GFAP, but not for S100B, in mild TBI (GSC 13-15) in patients with CT abnormalities. ${ }^{172}$ Results on the performance for these biomarkers vary between studies. Guidelines for the use of S100B in blood for the initial management of mild TBI have been published, ${ }^{173}$ with the aim to reduce the number of unnecessary CT scans in patients at low risk for intracranial complications (and possible neurosurgical interventions). These guidelines were recently validated in a large multicenter study on mild TBI, with analyses performed using a commercial assay on a fully automated instrument, showing a sensitivity of $97 \%$ and a specificity of $34 \%$ to identify intracranial hemorrhage on CT scans. ${ }^{174}$

SNTF is measurable in blood samples and show an increase after TBI, also in CT-negative mild TBI cases 175, 176, and has been suggested as a predictive marker in sports-related concussion ${ }^{177}$. Standard enzyme-linked immunosorbent assays for T-tau and NF-L were recently transferred onto the Single molecule array (Simoa) platform, which allows for the ultrasensitive measurement of these two proteins in the blood ${ }^{178,179}$. Plasma T-tau concentrations correlate poorly with CSF concentrations ${ }^{180}$, but in acute hypoxic brain injury the rise in plasma T-tau is predictive of outcome ${ }^{178}$. During the acute phase of severe TBI, increases in serum T-tau and P-tau have also been described, with levels decreasing, but not down to control levels, during the period up to six months following the acute phase ${ }^{150}$. In sports-related concussion, plasma T-tau has been found to predict return-to-play time with high accuracy ${ }^{181}$. Serum NF-L correlates strongly with CSF NF-L and increases over time in American football athletes over the course of a season ${ }^{182}$. High S100B levels in serum after TBI correlate with intracranial lesions detected using CT scan. ${ }^{183}$ For this reason, S100B in serum has been suggested as a fluid biomarker for acute TBI that could reduce the number of unnecessary CT scans of the brain in new clinical guidelines for the management of TBI ${ }^{183}$, However, the performance of S100B in serum to identify minor brain injury in mild TBI cases is suboptimal ${ }^{148}$.

\section{[H1]Management}

\section{[H2] Acute TBI Management}

Beginning at the scene of injury and in the emergency center, urgent assessment of risk for an intracranial hematoma or brain swelling includes a brief interview and neurological examination including the GCS to determine whether a CT scan of the brain is indicated ${ }^{184,}$ 
185. Observation for 6-8 $\mathrm{h}$ in the emergency center and repeat assessment may be an alternative to CT, e.g. in patients with high GCS scores and no focal neurological signs. Patient and caregiver education, including verbal and written instructions for identifying complications, observation by an adult for $24 \mathrm{~h}$, a regimen of rest (see below), and gradual resumption of activities is the standard of care at many centers.

In PCS cases with persistent symptoms, headaches persisting >3 months, especially the migraine type, may respond to medication ${ }^{186}$. Depression is common secondary to complicated mild $\mathrm{TBI}^{187}$, and can be treated by a combination of medication and behavioral approaches. Instructions for good sleep hygiene are recommended because sleep disturbance is also a frequent sequel of $\mathrm{mTBI}^{185}$.

The early post-injury period is associated with release of excitotoxic neurotransmitters, a neurometabolic crisis due to increased energy demand despite reduced cerebral blood flow, inflammation, and axonal dysfunction ${ }^{188}$. A second experimental mild TBI prior to resolution of the pathophysiological changes following the first injury may add cumulative damage and prolong recovery from the second injury as compared with spacing the two injuries further apart to allow the brain to recover sufficiently from the initial mild TBI. ${ }^{189}$, ${ }^{190}$ A case study showed evidence of ongoing neuronal damage in a knocked-out boxer for a period of several months. ${ }^{191}$ This window of vulnerability to re-injury has been cited as the rationale for prescribing rest for sports players who experience head trauma ${ }^{3}$ (Figure 5).

When the athlete becomes asymptomatic at rest, light exercise and cognitive activity are resumed and gradually increased while monitoring symptom level. If symptoms return after exertion, the level of activity is reduced. Otherwise, the athlete proceeds to resumption of non-contact training followed by return to contact practice and competitive play. Time from injury to return to play (RTP) is 7 to 10 days in $85 \%$ of collegiate athletes recovering from their first concussion. However, variability is considerable as RTP can be delayed for a month or longer ${ }^{3,192}$. Indeed, a first case report on a knocked-out boxer suggested that the time period with high CSF NF-L levels as a sign of ongoing axonal damage and dysfunction was extended over several months, ${ }^{191}$ and much longer than previously envisioned. Concomitant injuries to other body regions and depression can also delay RTP. Rest is also a frequent recommendation by emergency care physicians to patients recovering from a mild TBI due to external causes other than sports. However, 
clinical management and follow-up of patients treated for mild TBI varies depending on factors such as emergency department's policy, the treating physician, and identification of risk factors for delayed recovery. Concerns about this approach to concussion management include the lack of precise definitions of rest, ambiguities in its implementation, and evidence that durations of rest longer than 2-3 days may be deleterious ${ }^{193}$. To this point, translational research indicates that gradual introduction of exercise promotes neuroplasticity after concussion ${ }^{194}$.

\section{[H2]Persistent symptoms of CTE}

With sparse prospective, longitudinal studies of athletes exhibiting persistent symptoms associated with repeat sport-related concussion and/or repetitive head impacts; there is a lack of evidenced-based guidelines for either diagnosis or clinical management. Thus, current knowledge on clinical decision-making relies on retrospective clinical histories in neuropathologically confirmed cases of $\mathrm{CTE}^{102}$.

For development of late life symptoms of mood and cognitive impairment, age of the athlete in relation to the typical playing career duration in the sport may be relevant ${ }^{195}$. Further, the position played by the athlete and the number of years played as a professional and in college provides an index of exposure to repetitive head impacts that may correlate with biomarker evidence of brain injuries ${ }^{182}$. More detailed neurological and neuropsychological assessments together with MRI using advanced sequences may identify evidence of brain damage that may guide whether to consider recommending an athlete to retire from the sport.

In general, after taking into consideration the aforementioned factors, many clinicians would advise an athlete to retire from contact sports after sustaining multiple concussions especially if the injuries were temporally contiguous, i.e. within a single season. However, the evidence base for reaching this decision is weak and there is concern that long exposure to repetitive head impacts is sufficient to initiate a tau-based neurodegenerative process that is self-perpetuating. With a latent period estimated to be 8 to 10 years, the initial, pre-clinical stage of CTE is thought to be undetected by conventional imaging or clinical assessments ${ }^{196}$. The dilemma for the clinician and athlete is that current evidence does not permit estimation of the threshold of exposure for initiating this neurodegenerative process. Moreover, individual differences in genotype, medical and 
social history, and other vulnerabilities may predispose to CTE. Recently developed tau ligands for PET imaging in Alzheimer's disease can estimate the burden of tau in cerebral cortex, but validation of this technique in individuals at high risk for CTE is pending. In concert with other brain imaging modalities, fluid biomarkers, and monitoring neurobehavioral changes, it may soon be possible to identify CTE in a preclinical stage.

\section{[H1]Quality of life}

Life satisfaction following TBI may vary over time and is associated with multiple factors. Due to a dose-response relationship, levels of cognitive and physical disability are related to injury severity. ${ }^{197}$ However, besides injury severity, contextual factors such as income, mood, fatigue and social participation predict outcome too. ${ }^{198,199}$

Shortly post injury patients typically report low life satisfaction with a gradual improvement over the years. Locus of control, or the degree to which individuals believe that their health is controlled by factors within the person or external factors (outside the person), is related to health related quality of life after TBI (HR-QoL). ${ }^{200}$ An external oriented locus of control is negatively related to HR-QoL. Locus of control, self-efficacy and coping are intertwined concepts that may serve as potential targets to improve HRQoL. 12 months after mild TBI, symptoms and quality of life are similar to that of controls. $^{201}$ However, over time a loss of life roles and prevalence of depression may hamper such an improvement or even cause a further decline of life satisfaction. Because the consequences of TBI may vary with varying contexts between individuals, insight into the impact of TBI as experienced by the patient, is required. Further, people with serious disabilities may report high satisfaction with life whereas caregivers and clinicians may question their quality of life. A lack of awareness but also a re-evaluation of what matters in life by the patient, may explain such a different perspective.

\section{[H1]Outlook}

Despite considerable advances, research on mild TBI and CTE is still in its infancy, with many gaps to be filled (Figure 6). These gaps range from improved knowledge on biophysical mechanisms, epidemiological data on the prevalence of CTE in contact sports athletes, as well as the precise molecular pathway from acute axonal injury to chronic and progressive neurodegeneration. 


\section{[H2]Mechanistic questions}

\section{[H3]Which trauma forces cause brain damage in TBI and trigger CTE?}

Axonal injury is likely the central mechanism in TBI, which is accompanied by other changes such as inflammation, energy crisis and oxidative stress. However, further research is needed to resolve why axons are differentially vulnerable to trauma compared with other structures, and if injured axons with failed axonal transport recover and restore normal transport function. Further, the relationship between the extent of axonal injury and recovery rate or development of PCS after a single mild TBI episode is not known. Similarly, little is known on how many, or how severe, repeated TBIs are needed to initiate the neurodegeneration of CTE. Further, it is uncertain whether blast-related TBI and CTE in military personnel is due to brain damage caused by the blast overpressure, or whether the blast wind gives a detrimental acceleration of the head with resulting axonal shearing ${ }^{202}$. ENREF_194To resolve these questions, we need tools to quantify both the trauma forces and the neuronal damage in mild TBI and blast injuries.

\section{[H3]What governs recovery after acute concussion?}

Why a proportion of patients develop long-lasting PCS is unclear ${ }^{203}$. These patients might have more severe neuronal damage, or sustain damage related to the type of impact and localization, but the possible influences of psychogenic or psychosocial mechanisms remain to be examined.

\section{[H3]What is the risk for CTE among contact sports athletes?}

Although repetitive sub-concussive head impacts are thought to initiate the neurodegenerative process leading to $\mathrm{CTE}^{196}$, the number of known CTE cases is limited, and it is not known what percentage of the very high number of individuals participating in contact sports will develop CTE. Thus, longitudinal epidemiological studies in contact sports athletes are needed to learn how frequent CTE is, as well as its relation to number and severity of concussions, length of career and similar risk factors. Furthermore, it will also be important to examine which factors govern resiliency to CTE and resolve the possible influence on CTE risk of psychosocial factors such as alcohol and drug abuse, obesity, diabetes and hypertension.

\section{[H3]What is the role of CTE pathology and does it propagate in the brain?}


Recent preclinical discoveries suggest that repetitive concussive and/or sub-concussive brain injuries initiate tau pathology that then spreads to adjacent neurons in the absence of ongoing triggering factors ${ }^{204}$. However, we do not know if tau aggregates after TBI play a pathophysiological causal role in delayed neurodegeneration and development of CTE, or are they simply a marker of prior injury. Indeed, one neuropathology study on blast TBI in veterans report axonal, but no tau pathology, even in cases with long survival after blast, ${ }^{205}$ and another report distinct astroglial scarring with dense astrogliosis in several brain areas, but no or minimal tau pathology. ${ }^{206}$ Further studies, especially longitudinal biomarker and neuropathology studies, correlating different types of pathology in affected brain regions with disease stage in CTE cases, will be valuable to understand whether tau pathology is the end step (or bystander) in the history of dysfunctional neurons rather than the cause of symptoms, and whether CTE progression is due to stereotypic between-neuron spreading of tau pathology following neuronal networks, or whether the disease simply affects an increasing number of brain regions with increasing severity.

\section{[H3]What is the role of amyloid plaques in CTE?}

Involvement of brain A $\beta$ mis-metabolism and aggregation in after TBI is supported by animal experimental studies on rotational TBI showing sequential accumulation of APP, up regulation of the secretases and $A \beta$ generation with formation of diffuse amyloid plaques $^{66,207}$. It is unclear how TBI induce $A \beta$ oligomerization and aggregation, if $A \beta$ oligomers cause toxicity in the setting of TBI, and whether $\mathrm{A} \beta$ plaques and intra-axonal $\mathrm{A} \beta$ depositions play pathophysiologically causal roles in the increased risk of Alzheimer's disease. Tools that include CSF measurements and amyloid PET are available to monitor APP and A $\beta$ metabolism and aggregation in patients. Application of these biomarkers in longitudinal studies will resolve the question whether $A \beta$ aggregation is involved in CTE pathogenesis, or whether it is a bystander of neuronal damage and degeneration.

\section{[H3]Can we find an objective measure for neuronal damage in mild TBI?}

Some argue that the term concussion should be abandoned due to its vague definition and variable symptoms without any clinically identifiable pathological substrate ${ }^{208}$. Accordingly, there is an unmet need for biomarkers to identify and monitor axonal damage in mild TBI. However, the most studied blood biomarker, S100B, shows poor specificity to predict intracranial pathology by CT in mild TBI with many cases with false positive S100B results ${ }^{209}$. Further, S100B is present in peripheral organs, e.g. adipose tissue and 
chondrocytes $^{210}$, making it responsive to peripheral injuries and multi-trauma, further limiting its clinical usefulness. Given that the central mechanism in TBI is axonal damage, fluid biomarkers reflecting this type of damage may be useful. Ultrasensitive techniques to measure the axonal proteins $\operatorname{tau}^{178,181}$ and $\mathrm{NFL}^{179}, 182$ in blood samples have been developed, but these biomarkers need evaluation in further clinical studies.

\section{[H3]Can we find tools to identify CTE in the clinic?}

Symptoms associated with CTE may be difficult to differentiate not only from Alzheimer's disease and Parkinson's disease but also from PCS and depression in younger patients ${ }^{211}$. Thus, both from a clinical perspective and for clinical studies on CTE pathogenesis, diagnostic tools are highly desirable. For Alzheimer's disease, a number of biomarkers are by hand, including amyloid PET and CSF A $\beta 42$, both reflecting cortical $\beta$-amyloid deposition $^{212}$, the CSF biomarkers total tau and NFL reflecting damage to neuronal axons and phosphorylated tau protein that correlates with amount of cortical tau pathology ${ }^{141}$. In addition, recent developments have resulted in promising ligands for tau PET imaging, to visualize tau pathology directly in patients ${ }^{213}$. Given the similarity in pathology between Alzheimer's disease and CTE, these biomarkers are top candidate CTE markers, but there are as yet no clinical biomarker studies on patients with suspected CTE pathology.

\section{[H2]Prevention}

Guidelines for return to play have been proposed by the American Academy of Neurology $\mathrm{y}^{214}$. These state that a concussed athlete should not return to play with persistent post-concussion symptoms at rest or with exertion, reduced academic or sports performance, abnormal neurological exam, neuropsychological test findings, or CT/MRI findings indicating increased risk $^{214}$. Strict compliance with these guidelines by field and ringside physicians may reduce both short and long term suffering for contact sport athletes.

Following international consensus criteria on concussion in sports, an athlete diagnosed with concussion should not be allowed by the physician or other licensed health provider to return to play on the day of injury ${ }^{3}$. Due to the nature of the sport, this is not easily applied in boxing, where an alternative to secure the well being of the contenders is to introduce rules not allowing head blows. Further, the Word Medical Association (WMA) 
recommended the general ban of boxing in 2005, ${ }^{215}$ based on the risk for brain damage and that the basic intent of boxing is to knock the opponent unconscious. However, the liability lies on the shoulders of sports organizations and governments to regulate rules in a way that minimizes risk for chronic brain damage for athletes, especially for those who are underaged.

\section{[H2]Emerging treatments}

Factors such as the marked phenotypic heterogeneity (anatomic, physiologic, and genetic) across injury severity, and limitations of current outcome assessment tools, have hindered the development of new therapies in $\mathrm{TBI}^{216}$. Furthermore, successful translation of new therapies to patients has been problematic despite the implementation of carefully designed multi-center randomized controlled trials ${ }^{217-219}$; accordingly, new clinical trial design, such as comparative effectiveness, might help to define optimal care and facilitate successful $\mathrm{RCTs}^{220}$. Despite these challenges, several novel therapies merit discussion.

Cerebral edema, with resultant intracranial hypertension, has always been a key therapeutic target for severe TBI, but recent evidence suggests that brain edema could play a part across the injury spectrum ${ }^{221}$. Treatment of severe brain edema has traditionally been reactionary-via cerebrospinal fluid drainage, administration of osmolar agents, and/or craniectomy. Now, exploratory studies suggest new molecularly guided approaches to prevent its development. Emerging treatments such as glibenclamide targeting sulfonylurea receptor-1-mediated ion channel opening are in Phase II clinical trials (NCT01454154) ${ }^{222}$. Similarly, inhibition of aquaporin-4-mediated edema accumulation either directly or via blocking a cascade of events triggered by high mobility box-1 protein release and toll-like receptor activation is in pre-clinical testing ${ }^{223}$. Such approaches could improve care.

Regarding therapy for mild TBI, several approaches for treatment are under investigation. A recent report suggests that hyperthermia $\left(39^{\circ} \mathrm{C}\right)$ at the time of injury may magnify secondary damage and initiate degenerative processes such as CTE, with rapid normalization of body temperature being neuroprotective ${ }^{224}$. Further, recent pre-clinical studies suggest that acute accumulation and spread of a toxic cis-phosphorylated tau protein (cis P-tau) isomer may disrupt axonal microtubule networks and leas to apoptosis, while treatment with a monoclonal antibody against cis P-tau prevented its spread and improved outcome ${ }^{225}$. Last, cellular therapies may improve outcome by either aiding in the 
creation of new circuitry or expressing trophic factors that are neuroprotective or regenerative. A Phase I study of autologous BMMNCs in children with severe TBI suggested that this approach might be feasible, safe, and associated with reduced treatment intensity ${ }^{226}$.

\section{[H2]Fostering clinical research on TBI and CTE}

To improve our understanding of TBI and CTE pathogenesis, we need large longitudinal clinical studies combining clinical evaluations with genetic, imaging, biomarker and genetic data. As an example from other areas in clinical neuroscience, the Alzheimer's Disease Neuroimaging Initiative (ADNI) has had a vast impact on the understanding of Alzheimer's disease ${ }^{227}$. For acute TBI, the prospective Transforming Research and Clinical Knowledge in Traumatic Brain Injury (TRACK-TBI) study in the US (NCT02119182) and the Collaborative European NeuroTrauma Effectiveness Research in Traumatic Brain Injury (CENTER-TBI) study in Europe (NCT02210221) are two prospective observational studies across the TBI severity spectrum (including mild TBI) examines clinical, imaging, genetic and blood biomarkers in the acute phase with longitudinal clinical follow-up, with the goals to improve methods for diagnosis and prognosis, refine outcome assessment and TBI care. For CTE, the Understanding Neurologic Injury and Traumatic Encephalopathy (UNITE) $\operatorname{study}^{228}$ is a retrospective clinic-pathological study aiming at investigate the validity of recently proposed clinical criteria ${ }^{102}$ and to identify clinical features that improve prediction of CTE pathology, and the Diagnose CTE Research Project is a multicenter, prospective longitudinal study evaluating clinical criteria for the diagnosis of TES as well as fluid and imaging biomarkers for CTE with the aim to enable diagnosing CTE in living individuals. These, and other, clinical studies are likely to improve our understanding of the pathophysiology, and the ability to ide mithe 


\section{Box 1. Classification of traumatic brain injury}

\section{[H1]Mild TBI}

- Normal structural brain imaging

- Loss of consciousness lasting 0-30 min

- Altered mental state lasting $\leq 24 \mathrm{~h}$

- $\leq 1$ day of post-trauma amnesia

- Glasgow Coma Scale ${ }^{99}$ score of $13-15^{\ddagger}$

\section{[H1]Moderate TBI}

- Normal or abnormal structural brain imaging

- Loss of consciousness lasting $30 \mathrm{~min}$ to $24 \mathrm{~h}$

- Altered mental state* lasting $>24 \mathrm{~h}$

- 1-7 days of post-trauma amnesia

- Glasgow Coma Scale ${ }^{99}$ score of 9-12*

\section{[H1]Severe TBI}

- Normal or abnormal structural brain imaging

- Loss of consciousness lasting $>24 \mathrm{~h}$

- Altered mental state* lasting $>24 \mathrm{~h}$

- $\quad>7$ days of post-trauma amnesia

- Glasgow Coma Scale ${ }^{99}$ score of $<9$

Classification presented by the Department of Veterans Affairs (VA) and the Department of Defense (DoD) concussion/mTBI Working Group ${ }^{2}$

Best score in the first $24 \mathrm{~h}$ after trauma. 


\section{Box 2. Conditions associated with TBI}

\section{[H1]Sub-concussive head trauma}

- Head traumas that do not result in any subjective or objective symptoms

- Whether repeated sub-concussive head traumas in contact sports add to the risk of developing CTE is currently unknown

\section{[H1] Repetitive mild TBI}

- Repeated episodes of concussion or mild TBI (Box 1)

- Primarily experienced by contact sports athletes and in military personnel exposed to combat

- Believed to initiate the self-propagating chronic neurodegenerative disorder CTE

[H1] Post-concussive syndrome (PCS)

- Also known as post-concussion disorder (PCD)

- $\quad$ Mild TBI that persists beyond the expected recovery period (>3 months)

- Nonspecific subjective symptoms, such as headache, fatigue, dizziness, poor concentration, sleep disturbances, anxiety, irritability and depressed mood

- $\quad$ 10-15\% of individuals with concussion develop chronic symptoms

[H1] Chronic traumatic encephalopathy (CTE)

- Chronic neurodegenerative disorder initiated by repeated head trauma

- The clinical counterpart to CTE, while not fully defined, has been termed traumatic encephalopathy syndrome (TES)

- Both the clinical and pathological syndromes were first described in retired professional boxers and are now also recognized in athletes involved in many different contact sports and in military veterans 


\section{Box 3. Sports-related concussion}

Today, concussion in sports is diagnosed based on subjective symptoms. According to consensus diagnostic criteria, sports-related concussion $(\mathrm{SRC})^{3}$, is a "transient disturbance of brain function due to a sports-related blow to the head or other body region", causing acceleration of the head followed by onset of post-concussion symptoms, including somatic, cognitive, and emotional domains. Although loss of consciousness occurs in $<10 \%$ of $\mathrm{SRCs}^{3}$, these injuries (and non-sports mild TBI) are often followed immediately (or within minutes to hours) by onset of one or more post-concussion symptoms, including headaches, dizziness, unstable balance, fatigue, nausea, blurred vision, sleep disturbance, and cognitive deficit such as memory or concentration problems. Symptoms of anxiety or depression may also emerge.

Many athletic programs in the United States conduct pre-season "baseline" assessments that measure self-reported symptoms, balance and cognitive performance on standard tests often administered using computers and interpreted in relation to normative data. Following SRC, the clinician repeats this assessment to identify and grade the severity of PCS and cognitive deficit relative to baseline. Post-concussion symptoms and cognitive performance are monitored while the athlete completes a concussion management program. Loss of consciousness and post-traumatic amnesia duration (if present), number and severity of acute post-concussion symptoms predict time for recovery, which is often measured by the interval until the player is cleared to return to play ${ }^{192}$.

Modifiers of the effects of concussion include age; young adolescents and children are at risk for slower recovery than college and professional athletes whose symptoms typically resolve within two weeks ${ }^{3}$. Prior SRC is considered to be a risk factor for prolonged recovery, but the evidence is mixed $^{3}$. Similarly, some but not all studies indicate that females have a longer trajectory for resolution of PCS than males ${ }^{3}$. Similar to non-sportsmTBI, pre-existing neuropsychiatric conditions may complicate recovery from SRC ${ }^{229}$. 


\section{Figure 1. Types of forces in mild TBI.}

The central mechanism for neuronal damage in mild traumatic brain injury (TBI) is acceleration and deceleration biomechanical forces that cause strain and shearing forces on the long axons in the brain, resulting in diffuse axonal injury. a | Linear (translational) acceleration occurs from forces making the head move in the anterior-posterior direction. $b$ | Another force is rotational (angular) acceleration, which result from forces making the head to rotate sideways. c | Finally, impact deceleration is when the head forcefully decelerates, e.g. when an athlete's head hits the ground. The figure represents schematic examples of different types of forces, while many TBIs are combinations of the above.

\section{Figure 2. Tau pathology in chronic traumatic encephalopathy.}

a | Tau pathology typically is strikingly irregular perivascular pattern (arrows) with dense patches located at the depths of the cortical sulci.

b | Higher magnification of A, showing PHF-tau immunoreactive perivascular NFTs (arrows).

\section{Figure 3. Traumatic axonal injury in TBI.}

Traumatic axonal injury involves a complex set of pathophysiological events, which have been partially characterized. Rapid mechanical stretch, for example, can result in microtubule disruption (the nature of which is unclear) and impairs axonal transport with varicosity formation. Membrane leakage can involve frank membrane rupture, transient poration, or loss of ion channel permeation selectivity. The net effects of these changes can be axonal disconnection and aberrant co-localization of amyloid-beta $(\mathrm{A} \beta)$ that is the main component of amyloid plaques in Alzheimer's disease, and is generated by sequential cleavage of amyloid precursor protein (APP) by the enzymes $\beta$-secretase (BACE) and presenilin 1 (PS1). Rapid mechanical stretch or injury can also induce a cascade beginning with membrane leakage and leading to intra-axonal calcium elevation, calpain- caspasemediated spectrin proteolysis, neurofilament compaction and axonal collapse.

\section{Figure 4. Diffusion Tensor Imaging for assessment of traumatic axonal injury}

A | After concussive or mild traumatic brain injury (TBI), abnormalities such as reduced diffusion anisotropy that were not detected using conventional MRI sequences (panels 1-3) were apparent using diffusion tensor imaging (DTI) (panel 4). Red arrows point to areas of 
reduced anisotropy, indicated by less bright signal compared to the contralateral side. From Arfanakis et al American Journal of Neuroradiology 2002 fig 4.

B | Radiological-pathological correlation between reduction in anisotropy and number of injured axons in a mouse model of TBI. From Brody et al Handbook of Clinical Neurology 2015 Fig 17.8. Varying severities of controlled cortical impact traumatic brain injury were performed in mice at $1.0,1.5$, and $2.0 \mathrm{~mm}$ depth from the cortical surface. 24 hours later, mice were scanned with DTI to assess changes in relative anisotropy in the pericontusional brain white matter region (y-axis) and then the number of amyloid precursor protein (APP) stained injured axons was counted in histological sections corresponding to the same peri-contusional brain white matter regions. This study found a linear relationship between decreased anisotropy and increased traumatic axonal injury.

C | Reduced relative anisotropy, indicated by less bright green color (red arrows), abnormalities detected using DTI (panels 1 and 2) in bilateral orbitofrontal white matter after blast-related concussive TBI in a US Military service member. These abnormalities were not detected with conventional T2 weighted MRI (panels 3 and 4) or CT (not shown). Color indicates primary direction of white matter tracts as detected by DTI: green indicates anterior-posterior, red indicates right-left, blue indicates rostral-caudal. From C.L. Mac Donald \& D.L. Brody, unpublished data.

D | High spatial and angular resolution DTI. using $1.25 \times 1.25 \times 1.25 \mathrm{~mm}$ voxels in a normal control subject without abnormalities. With high spatial resolution DTI, the individual white matter tracts can be more clearly distinguished from each other and from surrounding structures than with the lower spatial resolution $(2.5 \times 2.5 \times 2.5 \mathrm{~mm}$ voxels $)$ DTI used to produce the images in panels A and C. L Holleran and D.L. Brody, unpublished data.

\section{Figure 5. Repeated traumatic brain injuries and the window of vulnerability}

After traumatic brain injury, the brain has increased susceptibility for a new injury during the recovery phase, the "window of vulnerability". A second mild injury before full resolution of pathophysiological changes following the first injury may add cumulative damage and prolong recovery from the second injury as compared with spacing the two injuries apart during a prolonged period of rest. Top: two traumatic brain injuries separated by an extended period of rest with complete normalization of pathophysiological changes. Bottom: two traumatic brain injuries, where the second injury occurs during the phase of increased vulnerability with a prolonged recovery phase and incomplete normalization of 
pathophysiological brain changes. This window of vulnerability to new brain injuries is the rationale for recommending contact sport athletes and extended return-to-play period after a concussion.

\section{Figure 6. Unknowns on the clinical and pathological pathways to chronic traumatic} encephalopathy.

Contact sports athletes are exposed for repeated concussions and/or sub-concussive blows, but most often recover quickly from symptoms. In an unknown proportion of at-risk individuals, repeated brain injury episodes are believed to initiate a chronic neurodegenerative disease that following a lag-phase of several years after the end of the contact sport career presents in the form of progressive cognitive, psychiatric and neurological symptoms and at autopsy primarily a tauopathy. Several unknowns require elucidation: The number, temporal distribution, and severity of traumatic brain injuries needed to initiate the disease process (1); why some patients develop long-term symptoms called post-concussion syndrome (PCS), and whether this syndrome is related to moresevere neuronal damage or to early CTE pathology, and to which degree psychiatric predisposition and psychosocial factors contribute (2); whether clinically evident CTE symptoms are preceded by mild PCS or whether there is a clinically silent period (3); whether pathology slowly builds up at the multiple sites of brain damage, or whether it spreads from damaged to undamaged sites (4); and the degree to which ageing, other neurodegenerative pathologies, or other risk factors for brain pathology, contribute to the clinical presentation (5). 


\section{REFERENCES}

1. Cassidy, J.D. et al. Incidence, risk factors and prevention of mild traumatic brain injury: results of the WHO Collaborating Centre Task Force on Mild Traumatic Brain Injury. J Rehabil Med, 28-60 (2004).

2. Management of Concussion/m, T.B.I.W.G. VA/DoD Clinical Practice Guideline for Management of Concussion/Mild Traumatic Brain Injury. J Rehabil Res Dev 46, CP1-68 (2009).

3. McCrory, P. et al. Consensus statement on concussion in sport: the 4th International Conference on Concussion in Sport held in Zurich, November 2012. Br J Sports Med 47, 250-8 (2013).

4. Medicine, A.C.o.R. Definition of mild traumatic brain injury. J Head Trauma Rehab 8, 86-87 (1993).

5. West, T.A. \& Marion, D.W. Current recommendations for the diagnosis and treatment of concussion in sport: a comparison of three new guidelines. $J$ Neurotrauma 31, 159-68 (2014).

6. Meares, S. et al. The prospective course of postconcussion syndrome: the role of mild traumatic brain injury. Neuropsychology 25, 454-65 (2011).

7. Ponsford, J., Cameron, P., Fitzgerald, M., Grant, M. \& Mikocka-Walus, A. Longterm outcomes after uncomplicated mild traumatic brain injury: a comparison with trauma controls. J Neurotrauma 28, 937-46 (2011).

8. Silverberg, N.D. \& Iverson, G.L. Etiology of the post-concussion syndrome: Physiogenesis and Psychogenesis revisited. NeuroRehabilitation 29, 317-29 (2011).

9. Martland, H.S. Punch drunk. Journal of the American Medical Association 91, 1103-1107 (1928).

10. Millspaugh, J. Dementia pugilistica. US Naval Med Bull 35, 297-303 (1937).

11. Critchley, M. Punch-drunk syndromes: the chronic traumatic encephalopathy of boxers. Hommage a Clovis Vincent (ed). Maloine, Paris (1949).

12. Blennow, K., Hardy, J. \& Zetterberg, H. The neuropathology and neurobiology of traumatic brain injury. Neuron 76, 886-99 (2012).

13. Gandy, S. et al. Chronic traumatic encephalopathy: clinical-biomarker correlations and current concepts in pathogenesis. Mol Neurodegener 9, 37 (2014).

14. Corsellis, J.A., Bruton, C.J. \& Freeman-Browne, D. The aftermath of boxing. Psychol Med 3, 270-303 (1973).

15. Stein, T.D. et al. Beta-amyloid deposition in chronic traumatic encephalopathy. Acta Neuropathol 130, 21-34 (2015).

16. Omalu, B.I. et al. Chronic traumatic encephalopathy in a National Football League player. Neurosurgery 57, 128-34; discussion 128-34 (2005).

17. McKee, A.C. et al. The first NINDS/NIBIB consensus meeting to define neuropathological criteria for the diagnosis of chronic traumatic encephalopathy. Acta Neuropathol 131, 75-86 (2016).

18. Hyder, A.A., Wunderlich, C.A., Puvanachandra, P., Gururaj, G. \& Kobusingye, O.C. The impact of traumatic brain injuries: a global perspective. NeuroRehabilitation 22, 341-53 (2007).

19. Peeters, W. et al. Epidemiology of traumatic brain injury in Europe. Acta Neurochirurgica 157, 1683-1696 (2015).

20. Centers for Disease Control and Prevention. (National Center for Injury Prevention and Control; Division of Unintentional Injury Prevention, Atlanta, GA, 2015).

21. Maas, A.I., Stocchetti, N. \& Bullock, R. Moderate and severe traumatic brain injury in adults. Lancet Neurol 7, 728-41 (2008). 
22. Whiteneck, G.G., Cuthbert, J.P., Corrigan, J.D. \& Bogner, J.A. Risk of Negative Outcomes After Traumatic Brain Injury: A Statewide Population-Based Survey. $J$ Head Trauma Rehabil 31, E43-54 (2016).

23. Roozenbeek, B., Maas, A.I. \& Menon, D.K. Changing patterns in the epidemiology of traumatic brain injury. Nature Reviews Neurology 9, 231-236 (2013).

24. Coronado, V.G. et al. Trends in Sports- and Recreation-Related Traumatic Brain Injuries Treated in US Emergency Departments: The National Electronic Injury Surveillance System-All Injury Program (NEISS-AIP) 2001-2012. J Head Trauma Rehabil 30, 185-97 (2015).

25. Fleminger, S., Oliver, D., Lovestone, S., Rabe-Hesketh, S. \& Giora, A. Head injury as a risk factor for Alzheimer's disease: the evidence 10 years on; a partial replication. Journal of Neurology, Neurosurgery \& Psychiatry 74, 857-862 (2003).

26. Nordström, P., Michaëlsson, K., Gustafson, Y. \& Nordström, A. Traumatic brain injury and young onset dementia: A nationwide cohort study. Annals of Neurology 75, 374-381 (2014).

27. Plassman, B.L. et al. Documented head injury in early adulthood and risk of Alzheimer's disease and other dementias. Neurology 55, 1158-66 (2000).

28. Gardner, R.C. \& Yaffe, K. Epidemiology of mild traumatic brain injury and neurodegenerative disease. Molecular and Cellular Neuroscience 66, Part B, 75-80 (2015).

29. McCrory, P., Meeuwisse, W.H., Kutcher, J.S., Jordan, B.D. \& Gardner, A. What is the evidence for chronic concussion-related changes in retired athletes: behavioural, pathological and clinical outcomes? British Journal of Sports Medicine 47, 327-330 (2013).

30. King, A.I. Fundamentals of impact biomechanics: Part I--Biomechanics of the head, neck, and thorax. Annu Rev Biomed Eng 2, 55-81 (2000).

31. Young, L.A., Rule, G.T., Bocchieri, R.T. \& Burns, J.M. Biophysical mechanisms of traumatic brain injuries. Semin Neurol 35, 5-11 (2015).

32. Cloots, R.J., Gervaise, H.M., van Dommelen, J.A. \& Geers, M.G. Biomechanics of traumatic brain injury: influences of the morphologic heterogeneities of the cerebral cortex. Ann Biomed Eng 36, 1203-15 (2008).

33. McKee, A.C. et al. Chronic traumatic encephalopathy in athletes: progressive tauopathy after repetitive head injury. J Neuropathol Exp Neurol 68, 709-35 (2009).

34. Gennarelli, T.A. et al. Diffuse axonal injury and traumatic coma in the primate. Ann Neurol 12, 564-74 (1982).

35. Pellman, E.J., Viano, D.C., Tucker, A.M., Casson, I.R. \& Waeckerle, J.F. Concussion in professional football: reconstruction of game impacts and injuries. Neurosurgery 53, 799-812; discussion 812-4 (2003).

36. Walilko, T.J., Viano, D.C. \& Bir, C.A. Biomechanics of the head for Olympic boxer punches to the face. Br J Sports Med 39, 710-9 (2005).

37. Atha, J., Yeadon, M.R., Sandover, J. \& Parsons, K.C. The damaging punch. $\mathrm{Br}$ Med J (Clin Res Ed) 291, 1756-7 (1985).

38. Oppenheimer, D.R. Microscopic lesions in the brain following head injury. Journal of Neurology, Neurosurgery, and Psychiatry 31, 299 (1968).

39. Blumbergs, P.C. et al. Stalning af amyloid percursor protein to study axonal damage in mild head Injury. The Lancet 344, 1055-1056 (1994).

40. McKee, A.C., Daneshvar, D.H., Alvarez, V.E. \& Stein, T.D. The neuropathology of sport. Acta neuropathologica 127, 29-51 (2014). 
41. Oppenheimer, D.R. Microscopic lesions in the brain following head injury. $J$ Neurol Neurosurg Psychiatry 31, 299-306 (1968).

42. McKee, A.C. et al. The spectrum of disease in chronic traumatic encephalopathy. Brain 136, 43-64 (2013).

43. McKee, A.C. et al. TDP-43 proteinopathy and motor neuron disease in chronic traumatic encephalopathy. J Neuropathol Exp Neurol 69, 918-29 (2010).

44. Bieniek, K.F. et al. Chronic traumatic encephalopathy pathology in a neurodegenerative disorders brain bank. Acta Neuropathol (2015).

45. Smith, D.H., Hicks, R. \& Povlishock, J.T. Therapy Development for Diffuse Axonal Injury. J Neurotrauma (2013).

46. Blumbergs, P.C. et al. Staining of amyloid precursor protein to study axonal damage in mild head injury. Lancet 344, 1055-6 (1994).

47. Blumbergs, P.C. et al. Topography of axonal injury as defined by amyloid precursor protein and the sector scoring method in mild and severe closed head injury. J Neurotrauma 12, 565-72 (1995).

48. Smith, D.H. et al. Immediate coma following inertial brain injury dependent on axonal damage in the brainstem. J Neurosurg 93, 315-22 (2000).

49. Magnoni, S. et al. Tau elevations in the brain extracellular space correlate with reduced amyloid-beta levels and predict adverse clinical outcomes after severe traumatic brain injury. Brain 135, 1268-80 (2012).

50. Niogi, S.N. \& Mukherjee, P. Diffusion tensor imaging of mild traumatic brain injury. J Head Trauma Rehabil 25, 241-55 (2010).

51. Tang-Schomer, M.D., Johnson, V.E., Baas, P.W., Stewart, W. \& Smith, D.H. Partial interruption of axonal transport due to microtubule breakage accounts for the formation of periodic varicosities after traumatic axonal injury. Exp Neurol 233, 364-72 (2012).

52. Christman, C.W., Grady, M.S., Walker, S.A., Holloway, K.L. \& Povlishock, J.T. Ultrastructural studies of diffuse axonal injury in humans. J Neurotrauma 11, 17386 (1994).

53. Gentleman, S.M., Nash, M.J., Sweeting, C.J., Graham, D.I. \& Roberts, G.W. Betaamyloid precursor protein (beta APP) as a marker for axonal injury after head injury. Neurosci Lett 160, 139-44 (1993).

54. Stone, J.R. et al. Impaired axonal transport and altered axolemmal permeability occur in distinct populations of damaged axons following traumatic brain injury. Exp Neurol 190, 59-69 (2004).

55. Marmarou, C.R., Walker, S.A., Davis, C.L. \& Povlishock, J.T. Quantitative analysis of the relationship between intra- axonal neurofilament compaction and impaired axonal transport following diffuse traumatic brain injury. J Neurotrauma 22, 1066-80 (2005).

56. Grady, M.S. et al. The use of antibodies targeted against the neurofilament subunits for the detection of diffuse axonal injury in humans. J Neuropathol Exp Neurol 52, 143-52 (1993).

57. Stone, J.R., Singleton, R.H. \& Povlishock, J.T. Intra-axonal neurofilament compaction does not evoke local axonal swelling in all traumatically injured axons. Exp Neurol 172, 320-31 (2001).

58. Povlishock, J.T. \& Katz, D.I. Update of neuropathology and neurological recovery after traumatic brain injury. J Head Trauma Rehabil 20, 76-94 (2005).

59. Shitaka, Y. et al. Repetitive closed-skull traumatic brain injury in mice causes persistent multifocal axonal injury and microglial reactivity. J Neuropathol Exp Neurol 70, 551-67 (2011). 
60. Buki, A. \& Povlishock, J.T. All roads lead to disconnection?--Traumatic axonal injury revisited. Acta Neurochir (Wien) 148, 181-93 (2006).

61. Bennett, R.E. \& Brody, D.L. Array tomography for the detection of non-dilated, injured axons in traumatic brain injury. J Neurosci Methods 245, 25-36 (2015).

62. Blennow, K., de Leon, M.J. \& Zetterberg, H. Alzheimer's disease. Lancet 368, 387403 (2006).

63. Magnoni, S. \& Brody, D.L. New perspectives on amyloid-beta dynamics after acute brain injury: moving between experimental approaches and studies in the human brain. Arch Neurol 67, 1068-73 (2010).

64. Ikonomovic, M.D. et al. Alzheimer's pathology in human temporal cortex surgically excised after severe brain injury. Exp Neurol 190, 192-203 (2004).

65. Roberts, G.W., Gentleman, S.M., Lynch, A. \& Graham, D.I. beta A4 amyloid protein deposition in brain after head trauma. Lancet 338, 1422-3. (1991).

66. Smith, D.H. et al. Accumulation of amyloid beta and tau and the formation of neurofilament inclusions following diffuse brain injury in the pig. J Neuropathol Exp Neurol 58, 982-92 (1999).

67. Brody, D.L. et al. Amyloid-beta dynamics correlate with neurological status in the injured human brain. Science 321, 1221-4 (2008).

68. Schwetye, K.E. et al. Traumatic brain injury reduces soluble extracellular amyloidbeta in mice: a methodologically novel combined microdialysis-controlled cortical impact study. Neurobiol Dis 40, 555-64 (2010).

69. Olsson, A. et al. Marked increase of beta-amyloid(1-42) and amyloid precursor protein in ventricular cerebrospinal fluid after severe traumatic brain injury. $J$ Neurol 251, 870-6 (2004).

70. Smith, D.H. et al. Brain trauma induces massive hippocampal neuron death linked to a surge in beta-amyloid levels in mice overexpressing mutant amyloid precursor protein. Am J Pathol 153, 1005-10. (1998).

71. Chen, X.H. et al. Long-term accumulation of amyloid-beta, beta-secretase, presenilin-1, and caspase-3 in damaged axons following brain trauma. Am J Pathol 165, 357-71 (2004).

72. Tran, H.T., Laferla, F.M., Holtzman, D.M. \& Brody, D.L. Controlled Cortical Impact Traumatic Brain Injury in 3xTg-AD Mice Causes Acute Intra-Axonal Amyloid-beta Accumulation and Independently Accelerates the Development of Tau Abnormalities. J Neurosci 31, 9513-25 (2011).

73. Lambert, M.P. et al. Diffusible, nonfibrillar ligands derived from Abeta1-42 are potent central nervous system neurotoxins. Proc Natl Acad Sci U S A 95, 6448-53 (1998).

74. Walsh, D.M. \& Selkoe, D.J. A beta oligomers - a decade of discovery. $J$ Neurochem 101, 1172-84 (2007).

75. Shankar, G.M. et al. Amyloid-beta protein dimers isolated directly from Alzheimer's brains impair synaptic plasticity and memory. Nat Med 14, 837-42 (2008).

76. Cleary, J.P. et al. Natural oligomers of the amyloid-beta protein specifically disrupt cognitive function. Nat Neurosci 8, 79-84 (2005).

77. Walsh, D.M. et al. Naturally secreted oligomers of amyloid beta protein potently inhibit hippocampal long-term potentiation in vivo. Nature 416, 535-9 (2002).

78. Esparza, T.J. et al. Amyloid-beta oligomerization in Alzheimer dementia versus high-pathology controls. Ann Neurol 73, 104-19 (2013). 
79. Gotz, J., Chen, F., van Dorpe, J. \& Nitsch, R.M. Formation of neurofibrillary tangles in P3011 tau transgenic mice induced by Abeta 42 fibrils. Science 293, 1491-5 (2001).

80. Tran, H.T., Sanchez, L. \& Brody, D.L. Inhibition of JNK by a Peptide Inhibitor Reduces Traumatic Brain Injury-Induced Tauopathy in Transgenic Mice. $J$ Neuropathol Exp Neurol 71, 116-29 (2012).

81. Loane, D.J., Kumar, A., Stoica, B.A., Cabatbat, R. \& Faden, A.I. Progressive neurodegeneration after experimental brain trauma: association with chronic microglial activation. J Neuropathol Exp Neurol 73, 14-29 (2014).

82. Smith, C. Review: the long-term consequences of microglial activation following acute traumatic brain injury. Neuropathol Appl Neurobiol 39, 35-44 (2013).

83. Aguzzi, A., Barres, B.A. \& Bennett, M.L. Microglia: scapegoat, saboteur, or something else? Science 339, 156-61 (2013).

84. Kumar, A., Alvarez-Croda, D.M., Stoica, B.A., Faden, A.I. \& Loane, D.J. Microglial/macrophage polarization dynamics following traumatic brain injury. $J$ Neurotrauma (2015).

85. Helmy, A., Carpenter, K.L., Menon, D.K., Pickard, J.D. \& Hutchinson, P.J. The cytokine response to human traumatic brain injury: temporal profiles and evidence for cerebral parenchymal production. J Cereb Blood Flow Metab 31, 658-70 (2011).

86. Helmy, A. et al. Recombinant human interleukin-1 receptor antagonist in severe traumatic brain injury: a phase II randomized control trial. J Cereb Blood Flow Metab 34, 845-51 (2014).

87. Hall, E.D., Vaishnav, R.A. \& Mustafa, A.G. Antioxidant therapies for traumatic brain injury. Neurotherapeutics 7, 51-61 (2010).

88. Ji, J. et al. Lipidomics identifies cardiolipin oxidation as a mitochondrial target for redox therapy of brain injury. Nat Neurosci 15, 1407-13 (2012).

89. Loane, D.J. \& Kumar, A. Microglia in the TBI brain: The good, the bad, and the dysregulated. Exp Neurol (2015).

90. Davidson, J., Cusimano, M.D. \& Bendena, W.G. Post-Traumatic Brain Injury: Genetic Susceptibility to Outcome. Neuroscientist 21, 424-41 (2015).

91. Huang, Y., Weisgraber, K.H., Mucke, L. \& Mahley, R.W. Apolipoprotein E: diversity of cellular origins, structural and biophysical properties, and effects in Alzheimer's disease. J Mol Neurosci 23, 189-204 (2004).

92. Poirier, J. Apolipoprotein E in animal models of CNS injury and in Alzheimer's disease. Trends Neurosci 17, 525-30 (1994).

93. Verghese, P.B., Castellano, J.M. \& Holtzman, D.M. Apolipoprotein E in Alzheimer's disease and other neurological disorders. Lancet Neurol 10, 241-52 (2011).

94. Bennett, R.E. et al. Human apolipoprotein E4 worsens acute axonal pathology but not amyloid-beta immunoreactivity after traumatic brain injury in 3xTG-AD mice. J Neuropathol Exp Neurol 72, 396-403 (2013).

95. Zeng, S. et al. Prognostic value of apolipoprotein E epsilon4 allele in patients with traumatic brain injury: a meta-analysis and meta-regression. Genet Test Mol Biomarkers 18, 202-10 (2014).

96. Jordan, B.D. et al. Apolipoprotein E epsilon4 associated with chronic traumatic brain injury in boxing. JAMA 278, 136-40 (1997).

97. Kutner, K.C., Erlanger, D.M., Tsai, J., Jordan, B. \& Relkin, N.R. Lower cognitive performance of older football players possessing apolipoprotein E epsilon4. Neurosurgery 47, 651-7; discussion 657-8 (2000). 
98. Kim, J., Yoon, H., Basak, J. \& Kim, J. Apolipoprotein E in synaptic plasticity and Alzheimer's disease: potential cellular and molecular mechanisms. Mol Cells 37, 767-76 (2014).

99. Teasdale G, J.B. Assessment of coma and impaired consciousness. A practical scale. Lancet Neurol 2, 81-84 (1974).

100. Teasdale G, M.A., Lecky F, Manley G, Stocchetti N, and Murray G. The Glasgow Coma Scale at 40 years: standing the test of time. Neurology 13, 844-854 (2014).

101. Theadom, A. et al. Persistent problems 1 year after mild traumatic brain injury: a longitudinal population study in New Zealand. Br J Gen Pract 66, e16-23 (2016).

102. Montenigro, P.H. et al. Clinical subtypes of chronic traumatic encephalopathy: literature review and proposed research diagnostic criteria for traumatic encephalopathy syndrome. Alzheimers Res Ther 6, 68 (2014).

103. Schneider, H.J., Kreitschmann-Andermahr, I., Ghigo, E., Stalla, G.K. \& Agha, A. Hypothalamopituitary dysfunction following traumatic brain injury and aneurysmal subarachnoid hemorrhage: a systematic review. Jama 298, 1429-38 (2007).

104. Krahulik, D., Zapletalova, J., Frysak, Z. \& Vaverka, M. Dysfunction of hypothalamic-hypophysial axis after traumatic brain injury in adults. $J$ Neurosurg 113, 581-4 (2010).

105. Hannon, M.J. et al. Acute glucocorticoid deficiency and diabetes insipidus are common after acute traumatic brain injury and predict mortality. $J$ Clin Endocrinol Metab 98, 3229-37 (2013).

106. Aimaretti, G. et al. Traumatic brain injury and subarachnoid haemorrhage are conditions at high risk for hypopituitarism: screening study at 3 months after the brain injury. Clin Endocrinol (Oxf) 61, 320-6 (2004).

107. Tanriverdi, F. \& Kelestimur, F. Neuroendocrine Disturbances after Brain Damage: An Important and Often Undiagnosed Disorder. J Clin Med 4, 847-57 (2015).

108. Klose, M. et al. Prevalence of posttraumatic growth hormone deficiency is highly dependent on the diagnostic set-up: results from The Danish National Study on Posttraumatic Hypopituitarism. J Clin Endocrinol Metab 99, 101-10 (2014).

109. Kelly, D.F. et al. Prevalence of pituitary hormone dysfunction, metabolic syndrome, and impaired quality of life in retired professional football players: a prospective study. J Neurotrauma 31, 1161-71 (2014).

110. Tanriverdi, F. et al. Kickboxing sport as a new cause of traumatic brain injurymediated hypopituitarism. Clin Endocrinol (Oxf) 66, 360-6 (2007).

111. Wilkinson, C.W. et al. High prevalence of chronic pituitary and target-organ hormone abnormalities after blast-related mild traumatic brain injury. Front Neurol 3, 11 (2012).

112. Tanriverdi, F. \& Kelestimur, F. Pituitary dysfunction following traumatic brain injury: clinical perspectives. Neuropsychiatr Dis Treat 11, 1835-43 (2015).

113. Klose, M. \& Feldt-Rasmussen, U. Hypopituitarism in Traumatic Brain Injury-A Critical Note. J Clin Med 4, 1480-97 (2015).

114. Brody, D.L., Mac Donald, C.L. \& Shimony, J.S. Current and future diagnostic tools for traumatic brain injury: CT, conventional MRI, and diffusion tensor imaging. Handb Clin Neurol 127, 267-75 (2015).

115. Yuh, E.L. et al. Magnetic resonance imaging improves 3-month outcome prediction in mild traumatic brain injury. Ann Neurol 73, 224-35 (2013).

116. Moenninghoff, C. et al. Diffuse axonal injury at ultra-high field MRI. PLoS One 10, e0122329 (2015). 
117. Hulkower, M.B., Poliak, D.B., Rosenbaum, S.B., Zimmerman, M.E. \& Lipton, M.L. A decade of DTI in traumatic brain injury: 10 years and 100 articles later. AJNR Am J Neuroradiol 34, 2064-74 (2013).

118. Mac Donald, C.L., Dikranian, K., Bayly, P., Holtzman, D. \& Brody, D. Diffusion tensor imaging reliably detects experimental traumatic axonal injury and indicates approximate time of injury. J Neurosci 27, 11869-76 (2007).

119. Mac Donald, C.L. et al. Detection of blast-related traumatic brain injury in U.S. military personnel. N Engl J Med 364, 2091-100 (2011).

120. Niogi, S.N. et al. Structural dissociation of attentional control and memory in adults with and without mild traumatic brain injury. Brain 131, 3209-21 (2008).

121. Magnoni, S. et al. Quantitative assessments of traumatic axonal injury in human brain: concordance of microdialysis and advanced MRI. Brain 138, 2263-77 (2015).

122. Nieuwenhuys, R., Voogd, J. \& van Huijzen, C. The Human Central Nervous System (Springer, 2008).

123. Grossman, E.J. et al. Thalamus and cognitive impairment in mild traumatic brain injury: a diffusional kurtosis imaging study. J Neurotrauma 29, 2318-27 (2012).

124. Morey, R.A. et al. Effects of chronic mild traumatic brain injury on white matter integrity in Iraq and Afghanistan war veterans. Hum Brain Mapp 34, 2986-99 (2013).

125. Presson, N. et al. An exploratory analysis linking neuropsychological testing to quantification of tractography using High Definition Fiber Tracking (HDFT) in military TBI. Brain Imaging Behav 9, 484-99 (2015).

126. Wang, X. et al. Diffusion basis spectrum imaging detects and distinguishes coexisting subclinical inflammation, demyelination and axonal injury in experimental autoimmune encephalomyelitis mice. NMR Biomed 27, 843-52 (2014).

127. Maudsley, A. et al. Distributions of MR Diffusion and Spectroscopy Measures with Traumatic Brain Injury. J Neurotrauma (2014).

128. Vagnozzi, R. et al. Assessment of metabolic brain damage and recovery following mild traumatic brain injury: a multicentre, proton magnetic resonance spectroscopic study in concussed patients. Brain 133, 3232-42 (2010).

129. Irimia, A. \& Van Horn, J.D. Functional neuroimaging of traumatic brain injury: advances and clinical utility. Neuropsychiatr Dis Treat 11, 2355-65 (2015).

130. Huang, M.X. et al. Single-subject-based whole-brain MEG slow-wave imaging approach for detecting abnormality in patients with mild traumatic brain injury. Neuroimage Clin 5, 109-19 (2014).

131. Tarapore, P.E. et al. Resting state magnetoencephalography functional connectivity in traumatic brain injury. J Neurosurg 118, 1306-16 (2013).

132. Huang, M.X. et al. Integrated imaging approach with MEG and DTI to detect mild traumatic brain injury in military and civilian patients. J Neurotrauma 26, 1213-26 (2009).

133. Lewine, J.D., Davis, J.T., Sloan, J.H., Kodituwakku, P.W. \& Orrison, W.W., Jr. Neuromagnetic assessment of pathophysiologic brain activity induced by minor head trauma. AJNR Am J Neuroradiol 20, 857-66 (1999).

134. Klunk, W.E. Amyloid imaging as a biomarker for cerebral beta-amyloidosis and risk prediction for Alzheimer dementia. Neurobiol Aging 32 Suppl 1, S20-36 (2011).

135. Hong, Y.T. et al. Amyloid imaging with carbon 11-labeled Pittsburgh compound B for traumatic brain injury. JAMA Neurol 71, 23-31 (2014). 
136. Scott, G. et al. Amyloid pathology and axonal injury after brain trauma. Neurology (2016).

137. Scholl, M., Damian, A. \& Engler, H. Fluorodeoxyglucose PET in Neurology and Psychiatry. PET Clin 9, 371-90, v (2014).

138. Byrnes, K.R. et al. FDG-PET imaging in mild traumatic brain injury: a critical review. Front Neuroenergetics 5, 13 (2014).

139. Okamura, N. et al. Advances in the development of tau PET radiotracers and their clinical applications. Ageing Res Rev (2016).

140. Villemagne, V.L., Fodero-Tavoletti, M.T., Masters, C.L. \& Rowe, C.C. Tau imaging: early progress and future directions. Lancet Neurol 14, 114-24 (2015).

141. Blennow, K., Hampel, H., Weiner, M. \& Zetterberg, H. Cerebrospinal fluid and plasma biomarkers in Alzheimer disease. Nat Rev Neurol 6, 131-44 (2010).

142. Csuka, E. et al. IL-10 levels in cerebrospinal fluid and serum of patients with severe traumatic brain injury: relationship to IL-6, TNF-alpha, TGF-beta1 and blood-brain barrier function. J Neuroimmunol 101, 211-21 (1999).

143. Kossmann, T. et al. Intrathecal and serum interleukin-6 and the acute-phase response in patients with severe traumatic brain injuries. Shock 4, 311-7 (1995).

144. Blennow, K. et al. No neurochemical evidence of brain injury after blast overpressure by repeated explosions or firing heavy weapons. Acta Neurol Scand 123, 245-51 (2011).

145. Zetterberg, H. et al. Neurochemical aftermath of amateur boxing. Arch Neurol 63, 1277-80 (2006).

146. Buttram, S.D. et al. Multiplex assessment of cytokine and chemokine levels in cerebrospinal fluid following severe pediatric traumatic brain injury: effects of moderate hypothermia. J Neurotrauma 24, 1707-17 (2007).

147. Kumar, R.G. et al. Acute CSF interleukin-6 trajectories after TBI: associations with neuroinflammation, polytrauma, and outcome. Brain Behav Immun 45, 253-62 (2015).

148. Zetterberg, H., Smith, D.H. \& Blennow, K. Biomarkers of mild traumatic brain injury in cerebrospinal fluid and blood. Nat Rev Neurol 9, 201-10 (2013).

149. Ost, M. et al. Initial CSF total tau correlates with 1-year outcome in patients with traumatic brain injury. Neurology 67, 1600-4 (2006).

150. Rubenstein, R. et al. A novel, ultrasensitive assay for tau: potential for assessing traumatic brain injury in tissues and biofluids. J Neurotrauma 32, 342-52 (2015).

151. Neselius, S. et al. CSF-biomarkers in Olympic boxing: diagnosis and effects of repetitive head trauma. PLoS ONE 7, e33606 (2012).

152. Olsson, B., Zetterberg, H., Hampel, H. \& Blennow, K. Biomarker-based dissection of neurodegenerative diseases. Prog Neurobiol (2011).

153. Bohmer, A.E. et al. Neuron-specific enolase, S100B, and glial fibrillary acidic protein levels as outcome predictors in patients with severe traumatic brain injury. Neurosurgery 68, 1624-30; discussion 1630-1 (2011).

154. Ross, S.A., Cunningham, R.T., Johnston, C.F. \& Rowlands, B.J. Neuron-specific enolase as an aid to outcome prediction in head injury. Br J Neurosurg 10, 471-6 (1996).

155. Ramont, L. et al. Effects of hemolysis and storage condition on neuron-specific enolase (NSE) in cerebrospinal fluid and serum: implications in clinical practice. Clin Chem Lab Med 43, 1215-7 (2005).

156. Czeiter, E. et al. Brain injury biomarkers may improve the predictive power of the IMPACT outcome calculator. J Neurotrauma 29, 1770-8 (2012). 
157. McMahon, P.J. et al. Measurement of the GFAP-BDP Biomarker for the Detection of Traumatic Brain Injury Compared to CT and MRI. J Neurotrauma (2014).

158. Raby, C.A. et al. Traumatic brain injury increases beta-amyloid peptide 1-42 in cerebrospinal fluid. J Neurochem 71, 2505-9 (1998).

159. Marklund, N. et al. Monitoring of beta-amyloid dynamics after human traumatic brain injury. J Neurotrauma 31, 42-55 (2014).

160. Riederer, B.M., Zagon, I.S. \& Goodman, S.R. Brain spectrin(240/235) and brain spectrin(240/235E): two distinct spectrin subtypes with different locations within mammalian neural cells. J Cell Biol 102, 2088-97 (1986).

161. Pike, B.R. et al. Accumulation of non-erythroid alpha II-spectrin and calpaincleaved alpha II-spectrin breakdown products in cerebrospinal fluid after traumatic brain injury in rats. J Neurochem 78, 1297-306 (2001).

162. Pineda, J.A. et al. Clinical significance of alphaII-spectrin breakdown products in cerebrospinal fluid after severe traumatic brain injury. J Neurotrauma 24, 354-66 (2007).

163. Farkas, O. et al. Spectrin breakdown products in the cerebrospinal fluid in severe head injury--preliminary observations. Acta Neurochir (Wien) 147, 855-61 (2005).

164. Mondello, S. et al. alphaII-spectrin breakdown products (SBDPs): diagnosis and outcome in severe traumatic brain injury patients. J Neurotrauma 27, 1203-13 (2010).

165. Wilkinson, K.D. et al. The neuron-specific protein PGP 9.5 is a ubiquitin carboxylterminal hydrolase. Science 246, 670-3 (1989).

166. Buki, A., Siman, R., Trojanowski, J.Q. \& Povlishock, J.T. The role of calpainmediated spectrin proteolysis in traumatically induced axonal injury. $J$ Neuropathol Exp Neurol 58, 365-75 (1999).

167. Diaz-Arrastia, R. et al. Acute biomarkers of traumatic brain injury: relationship between plasma levels of ubiquitin C-terminal hydrolase-L1 and glial fibrillary acidic protein. $J$ Neurotrauma 31, 19-25 (2014).

168. Nylen, K. et al. Increased serum-GFAP in patients with severe traumatic brain injury is related to outcome. J Neurol Sci 240, 85-91 (2006).

169. Okonkwo, D.O. et al. GFAP-BDP as an acute diagnostic marker in traumatic brain injury: results from the prospective transforming research and clinical knowledge in traumatic brain injury study. J Neurotrauma 30, 1490-7 (2013).

170. Papa, L. et al. Time Course and Diagnostic Accuracy of Glial and Neuronal Blood Biomarkers GFAP and UCH-L1 in a Large Cohort of Trauma Patients With and Without Mild Traumatic Brain Injury. JAMA Neurol 73, 551-60 (2016).

171. Welch, R.D. et al. Ability of Serum Glial Fibrillary Acidic Protein, Ubiquitin CTerminal Hydrolase-L1, and S100B To Differentiate Normal and Abnormal Head Computed Tomography Findings in Patients with Suspected Mild or Moderate Traumatic Brain Injury. J Neurotrauma 33, 203-14 (2016).

172. Metting, Z., Wilczak, N., Rodiger, L.A., Schaaf, J.M. \& van der Naalt, J. GFAP and S100B in the acute phase of mild traumatic brain injury. Neurology 78, 142833 (2012).

173. Unden, J., Ingebrigtsen, T., Romner, B. \& Scandinavian Neurotrauma, C. Scandinavian guidelines for initial management of minimal, mild and moderate head injuries in adults: an evidence and consensus-based update. BMC Med 11, 50 (2013).

174. Unden, L., Calcagnile, O., Unden, J., Reinstrup, P. \& Bazarian, J. Validation of the Scandinavian guidelines for initial management of minimal, mild and moderate traumatic brain injury in adults. BMC Med 13, 292 (2015). 
175. Siman, R. et al. A panel of neuron-enriched proteins as markers for traumatic brain injury in humans. J Neurotrauma 26, 1867-77 (2009).

176. Siman, R. et al. Evidence That the Blood Biomarker SNTF Predicts Brain Imaging Changes and Persistent Cognitive Dysfunction in Mild TBI Patients. Front Neurol 4, 190 (2013).

177. Siman, R. et al. Serum SNTF Increases in Concussed Professional Ice Hockey Players and Relates to the Severity of Post-Concussion Symptoms. J Neurotrauma (2014).

178. Randall, J. et al. Tau proteins in serum predict neurological outcome after hypoxic brain injury from cardiac arrest: results of a pilot study. Resuscitation 84, 351-6 (2013).

179. Gisslén, M. et al. Plasma concentration of the neurofilament light protein (NFL) is a biomarker of CNS injury in HIV infection: a cross-sectional study. EBioMedicine In press (2015).

180. Zetterberg, H. et al. Plasma tau levels in Alzheimer's disease. Alzheimers Res Ther 5, 9 (2013).

181. Shahim, P. et al. Blood biomarkers for brain injury in concussed professional ice hockey players. JAMA Neurol 71, 684-92 (2014).

182. Oliver, J. et al. Serum Neurofilament Light in American Football Athletes Over the Course of a Season. J Neurotrauma (2015).

183. Unden, J., Ingebrigtsen, T. \& Romner, B. Scandinavian guidelines for initial management of minimal, mild and moderate head injuries in adults: an evidence and consensus-based update. BMC Med 11, 50 (2013).

184. Jagoda AS, B.J., Bruns JJ Jr, et al, and the American College of Emergency Physicians, and the Centers for Disease Control and Prevention. Clinical policy: neuroimaging and decision making in mild traumatic brain injury in the acute setting. Ann Emerg Med 52 (2008).

185. Levin HS, D.-A.R. Diagnosis, prognosis, and clinical management of mild traumatic brain injury. Lancet Neurol 14, 506-17 (2015).

186. DiTommaso C, H.J., Lucas S, Dikmen S, Temkin N, Bell KR. Medication usage patterns for headache treatment after mild traumatic brain injury. Headache $\mathbf{5 4}$, 511-19 (2014).

187. Bombardier CH, F.J., Temkin NR, Esselman PC, Barber J, Dikmen SS. Rates of major depressive disorder and clinical outcomes following traumatic brain injury. JAMA 303, 1938-45 (2010).

188. Giza CC, H.D. The New Neurometabolic Cascade of Concussion. Neurosurgery 75, S24-S33 (2014).

189. Guskiewicz, K.M. et al. Cumulative effects associated with recurrent concussion in collegiate football players: the NCAA Concussion Study. JAMA 290, 2549-55 (2003).

190. Slobounov, S., Slobounov, E., Sebastianelli, W., Cao, C. \& Newell, K. Differential rate of recovery in athletes after first and second concussion episodes. Neurosurgery 61, 338-44; discussion 344 (2007).

191. Neselius, S., Brisby, H., Granholm, F., Zetterberg, H. \& Blennow, K. Monitoring concussion in a knocked-out boxer by CSF biomarker analysis. Knee Surg Sports Traumatol Arthrosc 23, 2536-9 (2015).

192. Meehan WP, M.R., Monuteaux MC, Stein CJ, Bachur RG. Early symptom burden predicts recovery after sport-related concussion. Neurology 83, 2204-10 (2014). 
193. Thomas, D., Apps, JN, Hoffmann, RG, McCrea, M, Hammeke, T. Benefits of strict rest after acute concussion: A randomized controlled trial. Pediatrics 135, 213-23 (2015).

194. Leddy JJ, C.J., Baker JG, Wack DS, Pendergast DR, Zivadinov R, Willer B. Exercise treatment for postconcussion syndrome: a pilot study of changes in functional magnetic resonance imaging activation, physiology, and symptoms. $J$ Head Trauma Rehabil 28, 241-9 (2013).

195. Montenigro, P.H. et al. Cumulative Head Impact Exposure Predicts Later-Life Depression, Apathy, Executive Dysfunction, and Cognitive Impairment in Former High School and College Football Players. J Neurotrauma (2016).

196. McKee, A.C. et al. The spectrum of disease in chronic traumatic encephalopathy. Brain 136, 43-64 (2013).

197. Willemse-van Son, A.H., Ribbers, G.M., Verhagen, A.P. \& Stam, H.J. Prognostic factors of long-term functioning and productivity after traumatic brain injury: a systematic review of prospective cohort studies. Clin Rehabil 21, 1024-37 (2007).

198. Grauwmeijer, E., Heijenbrok-Kal, M.H. \& Ribbers, G.M. Health-related quality of life 3 years after moderate to severe traumatic brain injury: a prospective cohort study. Arch Phys Med Rehabil 95, 1268-76 (2014).

199. Willemse-van Son, A.H., Ribbers, G.M., Hop, W.C. \& Stam, H.J. Community integration following moderate to severe traumatic brain injury: a longitudinal investigation. J Rehabil Med 41, 521-7 (2009).

200. Wielenga-Boiten, J.E., Heijenbrok-Kal, M.H. \& Ribbers, G.M. The Relationship of Health Locus of Control and Health-Related Quality of Life in the Chronic Phase After Traumatic Brain Injury. J Head Trauma Rehabil 30, 424-31 (2015).

201. Andelic, N. et al. Trajectories of physical health in the first 5 years after traumatic brain injury. J Neurol 262, 523-31 (2015).

202. Goldstein, L.E. et al. Chronic traumatic encephalopathy in blast-exposed military veterans and a blast neurotrauma mouse model. Sci Transl Med 4, 134ra60 (2012).

203. DeKosky, S.T., Blennow, K., Ikonomovic, M.D. \& Gandy, S. Acute and chronic traumatic encephalopathies: pathogenesis and biomarkers. Nat Rev Neurol 9, 192200 (2013).

204. Clavaguera, F., Goedert, M. \& Tolnay, M. [Induction and spreading of tau pathology in a mouse model of Alzheimer's disease]. Med Sci (Paris) 26, 121-4 (2010).

205. Ryu, J. et al. The problem of axonal injury in the brains of veterans with histories of blast exposure. Acta Neuropathol Commun 2, 153 (2014).

206. Shively, S.B. et al. Characterisation of interface astroglial scarring in the human brain after blast exposure: a post-mortem case series. Lancet Neurol 15, 944-53 (2016).

207. Johnson, V.E., Stewart, W. \& Smith, D.H. Traumatic brain injury and amyloid-beta pathology: a link to Alzheimer's disease? Nat Rev Neurosci 11, 361-70 (2010).

208. Sharp, D.J. \& Jenkins, P.O. Concussion is confusing us all. Pract Neurol 15, 17286 (2015).

209. Ingebrigtsen, T. et al. The clinical value of serum S-100 protein measurements in minor head injury: a Scandinavian multicentre study. Brain Inj 14, 1047-55 (2000).

210. Haimoto, H., Hosoda, S. \& Kato, K. Differential distribution of immunoreactive S100-alpha and S100-beta proteins in normal nonnervous human tissues. $L a b$ Invest 57, 489-98 (1987). 
211. Mez, J., Solomon, T.M., Daneshvar, D.H., Stein, T.D. \& McKee, A.C.

Pathologically Confirmed Chronic Traumatic Encephalopathy in a 25-Year-Old Former College Football Player. JAMA Neurol, 1-3 (2016).

212. Blennow, K., Mattsson, N., Scholl, M., Hansson, O. \& Zetterberg, H. Amyloid biomarkers in Alzheimer's disease. Trends Pharmacol Sci 36, 297-309 (2015).

213. Eisenmenger, L.B. et al. Advances in PET Imaging of Degenerative, Cerebrovascular, and Traumatic Causes of Dementia. Semin Nucl Med 46, 57-87 (2016).

214. Giza, C.C. et al. Summary of evidence-based guideline update: evaluation and management of concussion in sports: report of the Guideline Development Subcommittee of the American Academy of Neurology. Neurology 80, 2250-7 (2013).

215. http://www.wma.net/en/30publications/10policies/b6/. (2005).

216. Maas, A.I., Roozenbeek, B. \& Manley, G.T. Clinical trials in traumatic brain injury: past experience and current developments. Neurotherapeutics 7, 115-26 (2010).

217. Andrews, P.J. et al. Hypothermia for Intracranial Hypertension after Traumatic Brain Injury. N Engl J Med 373, 2403-12 (2015).

218. Nichol, A. et al. Erythropoietin in traumatic brain injury (EPO-TBI): a double-blind randomised controlled trial. Lancet 386, 2499-506 (2015).

219. Wright, D.W. et al. Very early administration of progesterone for acute traumatic brain injury. N Engl J Med 371, 2457-66 (2014).

220. Kochanek, P.M. et al. Operation Brain Trauma Therapy: Approach to Modeling, Therapy Evaluation, Drug Selection, and Biomarker Assessments for a Multicenter Pre-Clinical Drug Screening Consortium for Acute Therapies in Severe Traumatic Brain Injury. J Neurotrauma (2015).

221. Hasan, K.M. et al. Serial atlas-based diffusion tensor imaging study of uncomplicated mild traumatic brain injury in adults. $J$ Neurotrauma 31, 466-75 (2014).

222. Walcott, B.P., Kahle, K.T. \& Simard, J.M. Novel treatment targets for cerebral edema. Neurotherapeutics 9, 65-72 (2012).

223. Laird, M.D. et al. High mobility group box protein-1 promotes cerebral edema after traumatic brain injury via activation of toll-like receptor 4. Glia 62, 26-38 (2014).

224. Titus, D.J., Furones, C., Atkins, C.M. \& Dietrich, W.D. Emergence of cognitive deficits after mild traumatic brain injury due to hyperthermia. Exp Neurol 263, 25462 (2015).

225. Kondo, A. et al. Antibody against early driver of neurodegeneration cis P-tau blocks brain injury and tauopathy. Nature 523, 431-6 (2015).

226. Liao, G.P. et al. Autologous bone marrow mononuclear cells reduce therapeutic intensity for severe traumatic brain injury in children. Pediatr Crit Care Med 16, 245-55 (2015).

227. Weiner, M.W. et al. Impact of the Alzheimer's Disease Neuroimaging Initiative, 2004 to 2014. Alzheimers Dement 11, 865-84 (2015).

228. Mez, J. et al. Assessing clinicopathological correlation in chronic traumatic encephalopathy: rationale and methods for the UNITE study. Alzheimers Res Ther 7, 62 (2015).

229. Ponsford J, C.P., Fitzgerald M, Grant M, Mikocka-Walus A. Long-term outcomes after uncomplicated mild traumatic brain injury: a comparision with trauma controls. J Neurotrauma 28, 937-46 (2011). 
